\title{
$N$-Substituted-1,2,3-triazoles: synthesis, characterization and evaluation as cannabinoid ligands
}

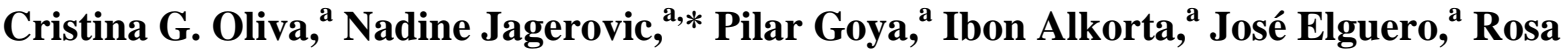 \\ Cuberes, ${ }^{b}$ and Alberto Dordal ${ }^{b}$ \\ a Instituto de Química Médica, CSIC, Juan de la Cierva 3, E-28006 Madrid, Spain \\ ${ }^{b}$ Laboratorios del Dr. Esteve S. A., Av. Mare de Déu de Montserrat, 221, E-08041 Barcelona, Spain \\ E-mail:nadine@iqm.csic.es
}

This paper is dedicated to Professor Benito Alcaide on the occasion of his $60^{\text {th }}$ anniversary

\begin{abstract}
A series of new N1-, N2- and N3-substituted 1,2,3-triazole derivatives has been synthesized by cycloaddition of butyltin azide with substituted alkynes followed by a $N$-alkylation reaction. The regioisomers have been isolated and characterized using NMR techniques. GIAO/B3LYP calculations of the absolute shieldings have been performed to verify the assignments and so the structures have been unequivocally identified. The proportion in which the three isomers are obtained corresponds with the relative order of stability indicated by the energy values calculated at the B3LYP level. CB1 cannabinoid receptor binding assays have been performed but none of the compounds showed significant activity.
\end{abstract}

Keywords: 1,2,3-Triazole, $N$-alkylation, cannabinoids

\section{Introduction}

The 1,2,3-triazole ring system has been the subject of considerable research mainly due to its usefulness in synthetic organic chemistry and also because of the pharmacological properties shown by some of its derivatives. In this context, we decided to explore the 1,2,3-triazole ring system as a new scaffold for cannabinoid ligands. ${ }^{1}$ Cannabinoids are compounds belonging to different structural families that elicit diverse biological responses by interacting with the cannabinoid receptors, of which two have been identified so far, CB1 and CB2. These receptors are involved in many biochemical processes and are thus interesting therapeutic targets. ${ }^{2-4}$ In particular, the $\mathrm{CB} 1$ receptor is involved in many different food-intake related disorders such as bulimia or obesity. ${ }^{5,6}$ Unfortunately, rimonabant (Figure 1), the first potent and selective CB1 antagonist to reach the pharmaceutical market as antiobesity agent, has been recently withdrawn 
due to possible depressive effects. ${ }^{7}$ Our group reported a series of cannabinoid 1,2,4-triazoles resulting in the identification of LH-21 (Figure 1) [5-(4-chlorophenyl)-1-(2,4-dichlorophenyl)-3hexyl-1H-1,2,4-triazole]. ${ }^{8}$ LH-21 displays selective and neutral CB1 receptor antagonism properties with lower penetration in the brain than rimonabant. ${ }^{9,10}$

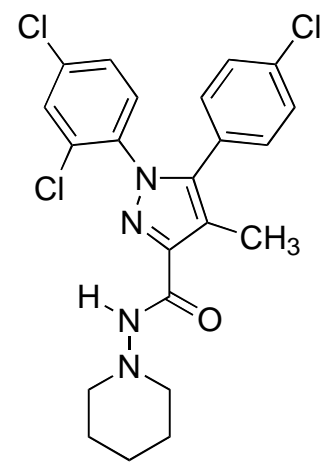

Rimonabant (SR141716A)
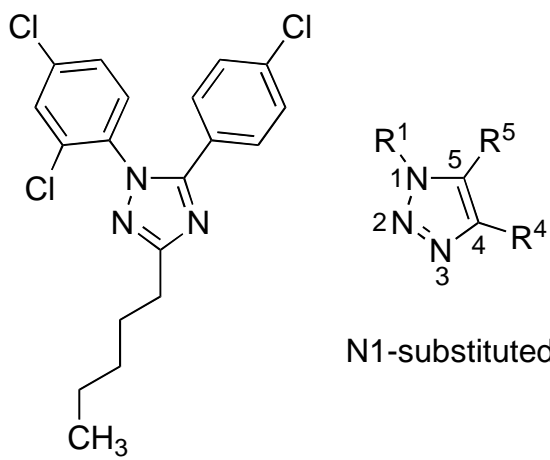

N1-substituted

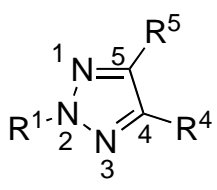

N2-substituted

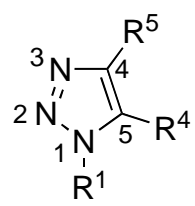

N3-substituted

LH-21

Figure 1. Structures of rimonabant and LH-21 together with the numbering of the isomers of $N$ substituted-1,2,3-triazoles.

On the basis of these findings, we have synthesized a series of 1,2,3-triazoles as LH-21 analogues. In the course of our research three reports dealing with cannabinoid 1,2,3-triazoles have appeared very recently. In one of them the 1,2,3-triazole group is reported as peptidomimetic element of mixed CB1/TRPV1 antagonists. ${ }^{11}$ The two other reports focused on 4-alkoxycarbonyl-1,5-diaryl-1,2,3-triazoles ${ }^{12}$ and 2-(phenoxy-carbonyl)methyl-1,2,3-triazoles ${ }^{13}$ as CB1 cannabinoid antagonists. The results reported here deal with the preparation of 2-alkyl1,2,3-triazoles as LH-21 analogues.

Concerning synthetic issues ${ }^{14}$ the most classical approach to the synthesis of 1,2,3-triazoles involves thermal 1,3-dipolar cycloaddition of azides with alkynes, as initially proposed by Huisgen. ${ }^{15}$ This reaction suffered from a lack of selectivity yielding a mixture of N1/N3- and N2substituted 1,2,3-triazoles when azides react with unsymmetrical disubstituted alkynes. ${ }^{16}$ The discovery of copper (I) ${ }^{17}$ and ruthenium (II) ${ }^{18}$ catalyzed cycloadditions opened the field of highly efficient "click chemistry" between azides and alkynes. However, using these conditions only N1/N3-substituted 1,2,3-triazole isomers can be prepared. Few methods are available for the selective preparation of N2-substituted-1,2,3-triazoles and they are limited to N2hydroxymethyl-, ${ }^{19} \mathrm{~N} 2$-allyl-, ${ }^{21,21}$ or N2-aryl-1,2,3-triazoles. ${ }^{22,23}$ We finally prepared and isolated the regioisomer N2-alkyl-1,2,3-triazoles by alkylation of $\mathrm{NH}$-1,2,3-triazoles. N2-[N-(piperidin-1yl)acetamide]-1,2,3-triazole derivatives are also reported here by comparison to rimonabant. The structural assignment of the different regioisomers was fully illustrated using NMR techniques. 


\section{Results and Discussion}

The synthesis of $\mathrm{NH}-1,2,3$-triazoles 2-6 was achieved in acceptable yield (30-67\%) by cycloaddition of tri-n-butyltin azide with mono- or disubstituted alkynes 1 under pressure and heating conditions (Scheme 1). ${ }^{24}$ This procedure was convenient regarding the safety issues using tri- $n$-butyltin azide compared to the highly explosive hydrazoic acid. The tributylstannyl group could be subsequently replaced by a proton under mild conditions. The starting alkynes 1a-1d were obtained from commercial sources and 1e was prepared ${ }^{25}$ in excellent yield from the corresponding 1-ethylnyl-4-methyl benzene.

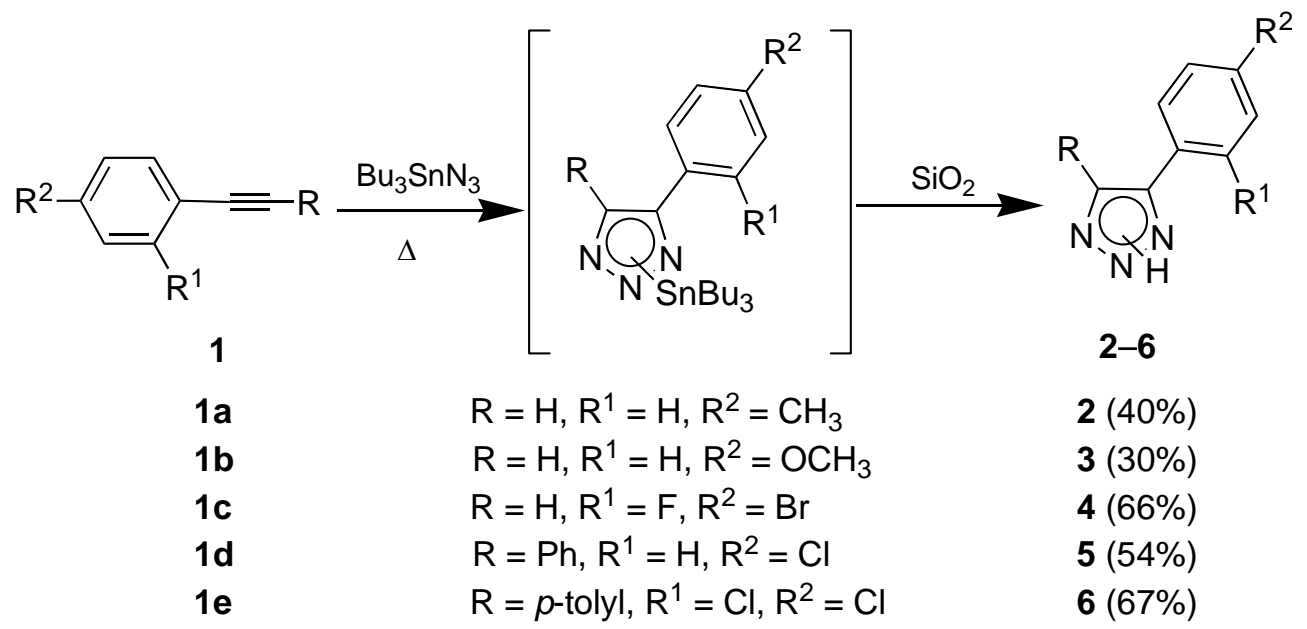

Scheme 1. Synthesis of $\mathrm{N} H-1,2,3$-triazoles 2-6.

Triazoles 2-6 were then alkylated with the corresponding alkylbromide under basic conditions using the phase transfer catalyst $\mathrm{Bu}_{4} \mathrm{NBr}^{26}$ to afford the desired $N$-substituted-1,2,3triazoles 7-17 (Scheme 2). $N$-Alkylation of unsymmetrical 4,5-disubstituted-1,2,3-triazoles produces a mixture of three possible regioisomers: a N2-, b N-1 and c N-3. Under the alkylation conditions used here two or three regioisomers have been isolated depending on the nature of the triazole substituents.

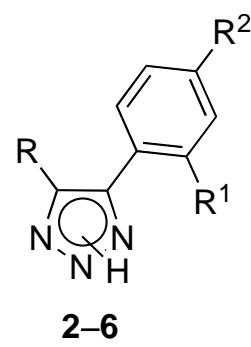

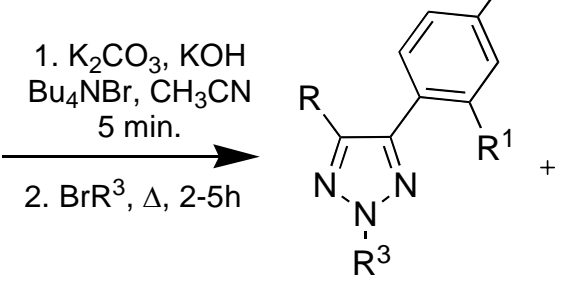

$7 a-17 a$

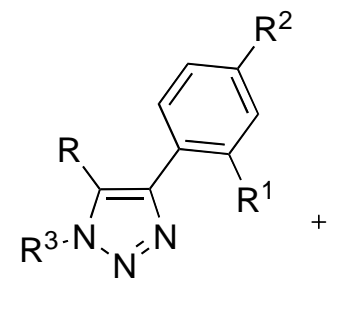

7b-17b

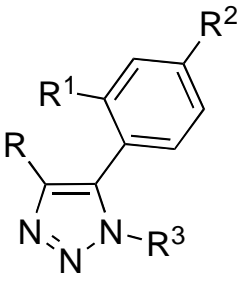

$7 c-17 c$

Scheme 2. Alkylation of 1,2,3-triazoles 2-6. 
The ratio of the regioisomers of 7-17, determined by ${ }^{1} \mathrm{H}$ NMR is reported in Table 1 . Each regioisomer has been isolated and fully identified by ${ }^{1} \mathrm{H},{ }^{13} \mathrm{C}, 2 \mathrm{D}-\mathrm{HSQC}$ and HMBC NMR data. The only 1,2,3-triazoles that have been characterized as a mixture of two isomers are 11b/11c and $\mathbf{1 7 b} / \mathbf{1 7} \mathbf{c}$.

Along the series 7-17, the regioisomer N2-substituted 1,2,3-triazole, a, was formed as the major product. The only exception came from the alkylation of 4-(4-bromo-2-fluorophenyl)1,2,3-triazole 4 with benzyl bromide that provided isomers 15a and 15b in a 45/55 ratio. It is interesting to note that the regioisomer N-3-substituted 1,2,3-triazole 7c-17c was formed in small proportion or was not detected. This fact probably is a consequence of steric factors such as suggested by the results obtained for 8, 9, 15 and 16. However, these factors are not valid for a higher proportion of isomer c over a in the alkylation of 7-14 and 17. This difference could be explained by a mesomeric effect of the benzene substituents.

Table 1. Synthesis of N-alkyl, benzyl, and ethoxycarbonyl-1,2,3-triazoles 7-17

\begin{tabular}{ccccccccc}
\hline $\mathrm{R}$ & $\mathrm{R}^{1}$ & $\mathrm{R}^{2}$ & $\mathrm{R}^{3}$ & $\mathrm{t}(\mathrm{h})$ & $\mathbf{a}(\%)^{\mathrm{a}}$ & $\mathbf{b}(\%)^{\mathrm{a}}$ & $\mathbf{c}(\%)^{\mathrm{a}}$ & $\mathbf{a} / \mathbf{b} / \mathbf{c}^{\mathrm{b}}$ \\
\hline $\mathrm{H}$ & $\mathrm{H}$ & $\mathrm{CH}_{3}$ & $n$-pentyl & 5 & $\mathbf{7 a}(62)$ & $\mathbf{7 b}(28)$ & $\mathbf{7 c}(5)$ & $65 / 29 / 6$ \\
$\mathrm{H}$ & $\mathrm{H}$ & $\mathrm{OCH}_{3}$ & $n$-pentyl & 5 & $\mathbf{8 a}(45)$ & $\mathbf{8 b}(25)$ & & $64 / 34 / 0$ \\
$\mathrm{H}$ & $\mathrm{F}$ & $\mathrm{Br}$ & $n$-pentyl & 5 & $\mathbf{9 a}(69)$ & $\mathbf{9 b}(29)$ & & $70 / 30 / 0$ \\
$\mathrm{Ph}$ & $\mathrm{H}$ & $\mathrm{Cl}$ & $n$-pentyl & 5 & $\mathbf{1 0 a}(50)$ & $\mathbf{1 0 b}(31)$ & $\mathbf{1 0 c}(9)$ & $55 / 35 / 10$ \\
$p$-tolyl & $\mathrm{Cl}$ & $\mathrm{Cl}$ & $n$-pentyl & 5 & $\mathbf{1 1 a}(73)$ & $\mathbf{1 1 b}$ & $\mathbf{1 1 c}^{\mathrm{c}}$ & $80 / 9 / 11$ \\
$\mathrm{Ph}$ & $\mathrm{H}$ & $\mathrm{Cl}$ & $n$-heptyl & 5 & $\mathbf{1 2 a}(64)$ & $\mathbf{1 2 b}(18)$ & $\mathbf{1 2 c}(9)$ & $70 / 20 / 10$ \\
$\mathrm{Ph}$ & $\mathrm{H}$ & $\mathrm{Cl}$ & $p$-Br-benzyl & 2 & $\mathbf{1 3 a}(62)$ & $\mathbf{1 3 b}(14)$ & $\mathbf{1 3 c}(19)$ & $65 / 15 / 20$ \\
$\mathrm{Ph}$ & $\mathrm{H}$ & $\mathrm{Cl}$ & benzyl & 2 & $\mathbf{1 4 a}(64)$ & $\mathbf{1 4 b}(24)$ & $\mathbf{1 4 c}(9)$ & $65 / 25 / 10$ \\
$\mathrm{H}$ & $\mathrm{F}$ & $\mathrm{Br}$ & benzyl & 2 & $\mathbf{1 5 a}(44)$ & $\mathbf{1 5 b}(54)$ & & $45 / 55 / 0$ \\
$\mathrm{H}$ & $\mathrm{F}$ & $\mathrm{Br}$ & $\mathrm{CH}_{2} \mathrm{CO}_{2} \mathrm{Et}$ & 5 & $\mathbf{1 6 a}(55)$ & $\mathbf{1 6 b}(18)$ & & $75 / 25 / 0$ \\
$\mathrm{Ph}$ & $\mathrm{H}$ & $\mathrm{Cl}$ & $\mathrm{CH}_{2} \mathrm{CO}_{2} \mathrm{Et}$ & 5 & $\mathbf{1 7 a}(60)$ & $\mathbf{1 7 b}$ & $\mathbf{1 7 c}^{\mathrm{c}}$ & $75 / 15 / 10$ \\
\hline
\end{tabular}

${ }^{a}$ Isolated yield after chromatography. ${ }^{b}$ determined by ${ }^{1} \mathrm{H}$ NMR of the reaction mixture. ${ }^{c}$ not isolated.

We have calculated at the B3LYP/6-311++G(d,p) level (see Computational details) the energies of the three isomers 10a $\left(-1360.77472\right.$ hartrees, $\left.E_{\text {rel }}=0.0 \mathrm{~kJ} \mathrm{~mol}^{-1}\right), \mathbf{1 0 b}\left(\mathrm{E}_{\text {rel }}=23.9 \mathrm{~kJ}\right.$ $\left.\mathrm{mol}^{-1}\right)$ and 10c $\left(\mathrm{E}_{\mathrm{rel}}=27.0 \mathrm{~kJ} \mathrm{~mol}^{-1}\right)$. The order of stability is the same that the proportion of isomers reported in Table $1(50 \%, 31 \%, 9 \%)$.

In order to illustrate the structural assignment of these regioisomers, 2D NMR studies of compound 10 are described here. 2D ${ }^{1} \mathrm{H}-{ }^{13} \mathrm{C}$ HMBC NMR analysis performed on the major regioisomer indicated the lack of correlations between the $\mathrm{H}-1$ ' signal of the pentyl chain $(\delta=$ $\left.4.36 \mathrm{ppm}, \mathrm{t},{ }^{3} J_{1^{\prime}, 2^{\prime}}=7.0 \mathrm{~Hz}, 2 \mathrm{H}\right)$ and the C-5 and C-4 signals of the 1,2,3-triazole ring $(\delta=144.2$ and $142.9 \mathrm{ppm}$ ) allowing the identification of 10a (Figure 2). On the spectra of the two other 
isolated regioisomers, 10b and 10c, these correlations were observed between the H-1' signal ( $\delta$ $\left.=4.17 \mathrm{ppm}, \mathrm{t},{ }^{3} J_{1^{\prime}, 2^{\prime}}=7.3 \mathrm{~Hz}, 2 \mathrm{H}\right)$ and $\mathrm{C}-5 \operatorname{signal}(\delta=133.7 \mathrm{ppm})$ for one of them and the H-1' signal $\left(\delta=4.18 \mathrm{ppm}, \mathrm{t},{ }^{3} J_{1^{\prime}, 2^{\prime}}=7.4 \mathrm{~Hz}, 2 \mathrm{H}\right)$ and the $\mathrm{C}-5$ signal $(\delta=132.4 \mathrm{ppm})$ for the other one (Figure 3) but they did not allow the identification of 10b and 10c. Furthermore, support for localization of the pentyl chain was provided on one hand by correlations between C-4 ( $\delta=$ $143.1 \mathrm{ppm})$ and $\mathrm{C}_{\text {para }}(\delta=133.3 \mathrm{ppm})$ signals with the $\mathrm{H}_{\text {ortho }}$ signal $\left(\delta=7.44 \mathrm{ppm}, \mathrm{dt}^{3}{ }^{3} J_{\mathrm{o}, \mathrm{m}}=8.6\right.$ $\mathrm{Hz},{ }^{4} J_{\mathrm{m}, \mathrm{m}}={ }^{5} J_{\mathrm{o}, \mathrm{m}}=2.2 \mathrm{~Hz}, 2 \mathrm{H}$ ) in the case of the regioisomer $10 \mathrm{~b}$ (Figure 3). On the other hand, correlations between $\mathrm{C}-5(\delta=132.4 \mathrm{ppm})$ and $\mathrm{C}_{\text {para }}(\delta=135.9 \mathrm{ppm})$ signals with $\mathrm{H}_{\text {ortho' }}$ signal $(\delta$ $=7.29-7.24 \mathrm{ppm}, \mathrm{m}, 5 \mathrm{H}$ ) were consistent with the regioisomer 10c (experimental data on Scheme 3).

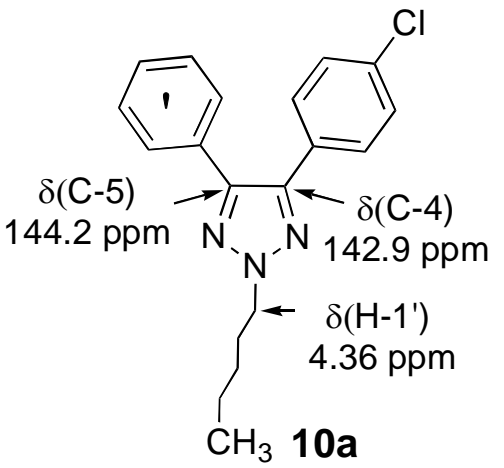

Figure 2. Lack of correlation between $\mathrm{H}-1$ ' and $\mathrm{C}-5$ and $\mathrm{C}-4$ signals of 10a from HMBC spectrum.
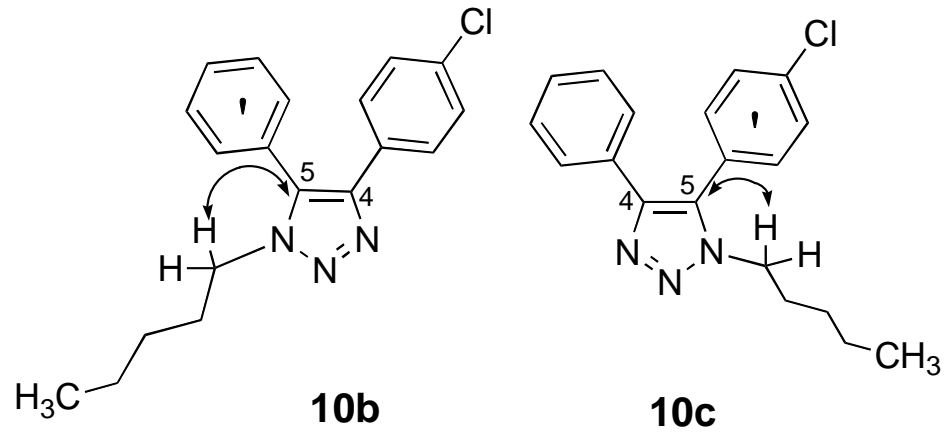

Figure 3. Correlation between $\mathrm{H}-1$ ' and C-5 signals of 10b and 10c (HMBC NMR spectrum).

We have carried out GIAO/ B3LYP/6-311++G(d,p) calculations of absolute shieldings on the three isomers to verify the assignments (see Computational details). First we have examined the ${ }^{13} \mathrm{C}$ chemical shifts. As we have shown in previous works, the carbon atoms bearing chlorine substituents are underestimated. ${ }^{27}$ Thus to compare the experimental chemical shifts $(\delta, \mathrm{ppm})$ with the calculated absolute shieldings $(\sigma, \mathrm{ppm})$ these three atoms were described with a dummy 
variable. The resulting equation is: $\delta^{13} \mathrm{C}=(176.4 \pm 0.4)-(0.987 \pm 0.004) \sigma^{13} \mathrm{C}-(8.5 \pm 0.9) \mathrm{CCl}, \mathrm{n}$ $=57, \mathrm{R}^{2}=0.999$. This equation, similar to those we have published, ${ }^{28}$ corresponds to the fitted values (in bold) for the carbon atoms of the triazole ring (the most sensitive to positional isomerism):
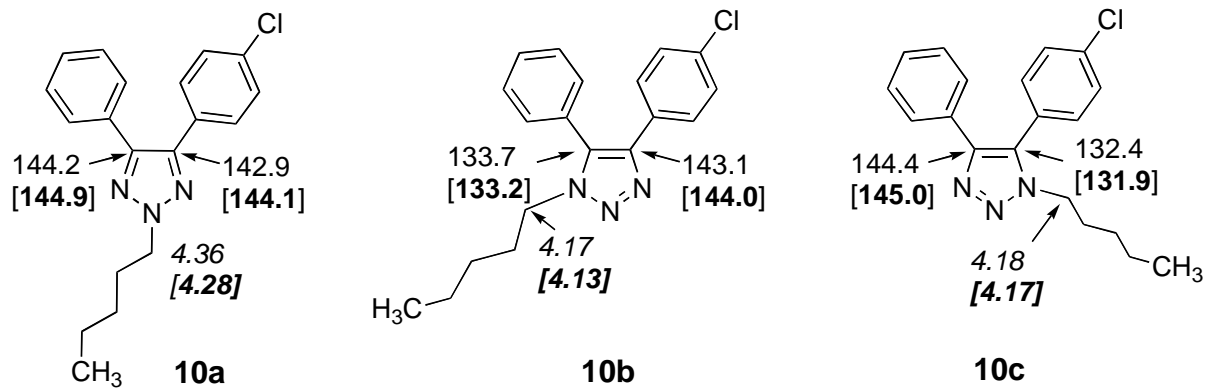

Scheme 3. Experimental and calculated (in bold) chemical shifts. ${ }^{1} \mathrm{H}$ data in italic.

Then, we have examined the ${ }^{1} \mathrm{H}$ NMR chemical shifts. In this case we have averaged the calculated values of isochronous protons by rotation $\left(\mathrm{H}_{\text {ortho }}, \mathrm{H}_{\text {para }}, \mathrm{CH}_{2}\right.$ and $\left.\mathrm{CH}_{3}\right)$. Using the assigned signals, we found $\delta^{1} \mathrm{H}=(31.0 \pm 0.4)-(0.970 \pm 0.013) \sigma^{1} \mathrm{H}, \mathrm{n}=27, \mathrm{R}^{2}=0.995$, identical to that we have observed for other compounds. ${ }^{29}$ The fitted values of the first methylene group (1') are given in Scheme 3.

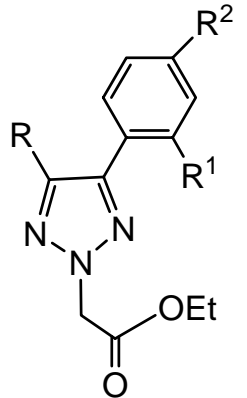

16a-17a

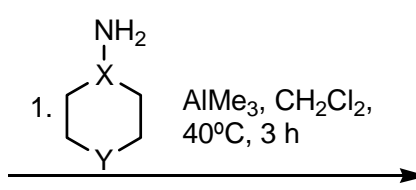

2. $\mathrm{HCl}(2 \mathrm{~N}), 40^{\circ} \mathrm{C}, 30 \mathrm{~min}$

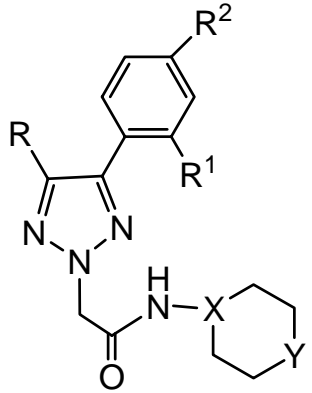

18-23

$$
\begin{array}{lll}
\text { 16a } & \mathrm{R}=\mathrm{Ph}, \mathrm{R}^{1}=\mathrm{H}, \mathrm{R}^{2}=\mathrm{Cl}, \mathrm{X}=\mathrm{N}, \mathrm{Y}=\mathrm{CH}_{2} & 1 \mathbf{1 8}(38 \%) \\
17 \mathbf{a} & \mathrm{R}=\mathrm{H}, \mathrm{R}^{1}=\mathrm{F}, \mathrm{R}^{2}=\mathrm{Br}, \mathrm{X}=\mathrm{N}, \mathrm{Y}=\mathrm{CH}_{2} & 19(44 \%) \\
16 \mathbf{a} & \mathrm{R}=\mathrm{Ph}, \mathrm{R}^{1}=\mathrm{H}, \mathrm{R}^{2}=\mathrm{Cl}, \mathrm{X}=\mathrm{N}, \mathrm{Y}=\mathrm{O} & \mathbf{2 0}(70 \%) \\
17 \mathrm{a} & \mathrm{R}=\mathrm{H}, \mathrm{R}^{1}=\mathrm{F}, \mathrm{R}^{2}=\mathrm{Br}, \mathrm{X}=\mathrm{N}, \mathrm{Y}=\mathrm{O} & 21(64 \%) \\
16 \mathrm{a} & \mathrm{R}=\mathrm{Ph}, \mathrm{R}^{1}=\mathrm{H}, \mathrm{R}^{2}=\mathrm{Cl}, \mathrm{X}=\mathrm{CH}, \mathrm{Y}=\mathrm{CH}_{2} & 22(56 \%) \\
17 \mathrm{a} & \mathrm{R}=\mathrm{H}, \mathrm{R}^{1}=\mathrm{F}, \mathrm{R}^{2}=\mathrm{Br}, \mathrm{X}=\mathrm{CH}, \mathrm{Y}=\mathrm{CH}_{2} & 23(42 \%)
\end{array}
$$

Scheme 5. 2-Acetamide-1,2,3-triazoles 18-23.

Carboxamides of related diaryl pyrazoles, such as rimonabant, are of particular interest in the field of cannabinoid ligands. Therefore we decided to synthesize 2-acetamide-1,2,3-triazoles 18- 
23. These were prepared starting from 2-ethoxycarbonylmethyl-1,2,3-triazoles 16a and 17a as described in Scheme 4. The esters 16a and 17a were treated with dimethylaluminum amides prepared from trimethylaluminum and the corresponding amine following a procedure previously used by us for conversion of ethyl esters to carboxylic acid hydrazides under mild conditions.

${ }^{1} \mathrm{H}$ and ${ }^{13} \mathrm{C}$ NMR data of 18-21 (Table 2) suggested the existence of two conformers. The fact that the amide $\mathrm{N}-\mathrm{C}$ bonds have a partial double-bond character causes a substantial rotational barrier that enables the $\operatorname{cis}(E)$-trans $(Z)$ interconversion resulting in magnetic non-equivalence between protons of the two rotamers. ${ }^{31}$ This $E-Z$ isomerism often plays an important role in receptor affinity due to possible hydrogen bonding patterns. Amides in solution show a preference for the $Z$-isomer that may be explained by possible steric interactions.

Table 2. ${ }^{1} \mathrm{H}$ NMR and ${ }^{13} \mathrm{C}$ NMR data and ratio of amide rotamers 18-21
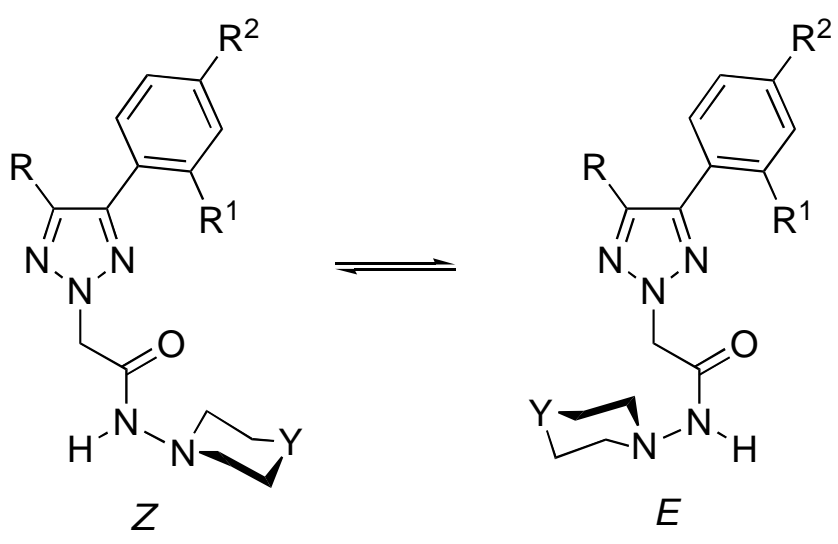

\begin{tabular}{|c|c|c|c|c|c|c|c|}
\hline \multirow{2}{*}{ Cpds } & \multirow{2}{*}{$\mathrm{M} / \mathrm{m}^{\mathrm{a}}$} & \multicolumn{2}{|c|}{${ }^{1} \mathrm{H}$ NMR $\delta(\mathrm{ppm})$} & \multicolumn{4}{|c|}{${ }^{13} \mathrm{C}$ NMR $\delta(\mathrm{ppm})$} \\
\hline & & $\mathrm{NH}$ & $\mathrm{CH}_{2} \mathrm{CO}$ & $\mathrm{CO}$ & $\mathrm{CH}_{2} \mathrm{CO}$ & $\mathrm{N}-\mathrm{CH}_{2} \mathrm{CH}_{2}$ & $\mathrm{~N}-\mathrm{CH}_{2}-\mathrm{CH}_{2}$ \\
\hline \multirow{4}{*}{18} & \multirow{4}{*}{$80 / 20$} & $9.35(\mathrm{~m})$ & $5.46(\mathrm{M})$ & 168.1 & 56.7 & $57.3(\mathrm{M})$ & $26.0(\mathrm{M})$ \\
\hline & & $9.03(\mathrm{M})$ & $5.08(\mathrm{~m})$ & (M) & (m) & $56.3(\mathrm{~m})$ & $25.8(\mathrm{~m})$ \\
\hline & & & & 162.8 & 56.2 & & \\
\hline & & & & $(\mathrm{m})$ & (M) & & \\
\hline \multirow{4}{*}{19} & \multirow{4}{*}{$80 / 20$} & $6.71(\mathrm{~m})$ & $5.45(\mathrm{M})$ & 167.9 & 57.2 & $58.1(\mathrm{M})$ & $25.6(\mathrm{M})$ \\
\hline & & $6.35(\mathrm{M})$ & $5.15(\mathrm{~m})$ & $(\mathrm{M})$ & $(\mathrm{m})$ & $56.8(\mathrm{~m})$ & $25.1(\mathrm{~m})$ \\
\hline & & & & 162.9 & 55.6 & & \\
\hline & & & & $(\mathrm{m})$ & (M) & & \\
\hline \multirow{4}{*}{20} & \multirow{4}{*}{$70 / 30$} & $9.54(\mathrm{~m})$ & $5.55(\mathrm{M})$ & 167.6 & 55.8 & $55.7(\mathrm{M})$ & $65.9(\mathrm{M})$ \\
\hline & & $9.15(\mathrm{M})$ & $5.13(\mathrm{~m})$ & $(\mathrm{M})$ & (m) & $54.7(\mathrm{~m})$ & $65.8(\mathrm{~m})$ \\
\hline & & & & 162.5 & 55.5 & & \\
\hline & & & & $(\mathrm{m})$ & (M) & & \\
\hline
\end{tabular}


Table 2. Continued

\begin{tabular}{cccccccc}
\hline \multirow{2}{*}{ Cpds } & \multirow{2}{*}{$\mathrm{M} / \mathrm{m}^{\mathrm{a}}$} & \multicolumn{2}{c}{$\mathrm{H} \mathrm{NMR} \delta(\mathrm{ppm})$} & \multicolumn{5}{c}{${ }^{13} \mathrm{C} \mathrm{NMR} \delta(\mathrm{ppm})$} \\
\cline { 3 - 7 } & $\mathrm{NH}$ & $\mathrm{CH}_{2} \mathrm{CO}$ & $\mathrm{CO}$ & $\mathrm{CH}_{2} \mathrm{CO}$ & $\mathrm{N}-\mathrm{CH}_{2} \mathrm{CH}_{2}$ & $\mathrm{~N}-\mathrm{CH}_{2}-\mathrm{CH}_{2}$ \\
\hline & & $9.45(\mathrm{~m})$ & $5.53(\mathrm{M})$ & 167.5 & 55.9 & $55.7(\mathrm{M})$ & $65.9(\mathrm{M})$ \\
& & $9.10(\mathrm{M})$ & $5.10(\mathrm{~m})$ & $(\mathrm{M})$ & $(\mathrm{m})$ & $54.7(\mathrm{~m})$ & $65.8(\mathrm{~m})$ \\
& & & & 162.4 & 55.6 & & \\
& & & $(\mathrm{~m})$ & $(\mathrm{M})$ & & \\
\hline
\end{tabular}

$\mathrm{M}=$ major conformer; $\mathrm{m}=$ minor conformer; ${ }^{\mathrm{a}}$ The ratio of the conformers has been determined by ${ }^{1} \mathrm{H}$ NMR.

The ${ }^{1} \mathrm{H}$ NMR spectra of 18-21 showed two signals for $\mathrm{NH}$ and two signals for $\mathrm{CH}_{2} \mathrm{CO}$. The rotamers ratios reported in Table 2 have been determined according to ${ }^{1} \mathrm{H}$ NMR signal integration. The ${ }^{13} \mathrm{C}$ NMR data of 18-21 were in agreement with the presence of these two rotamers with two signals for each of the following carbons: $\mathrm{CO}, \mathrm{CH}_{2}$ and piperidine- and morpholine-C-2' and C-3'. In the case of the cyclohexyl derivatives 22 and 23, no chemical shift differences have been shown in ${ }^{1} \mathrm{H}$ or in ${ }^{13} \mathrm{C}$ NMR spectra.

These ratios correspond, respectively, to $3.4\left(\mathrm{Y}=\mathrm{CH}_{2}\right)$ and $2.1 \mathrm{~kJ} \mathrm{~mol}^{-1}(\mathrm{Y}=\mathrm{O})$. To assign the $E / Z$ isomers to the $\mathrm{M} / \mathrm{m}$ ones, we have carried out calculations at the B3LYP/6-311++G(d,p) level of the energies and these minimized geometries of absolute shieldings [GIAO/ B3LYP/6$311++\mathrm{G}(\mathrm{d}, \mathrm{p})]$. In all cases, the $Z$ isomers are more stable that the $E$ ones by about $14 \mathrm{~kJ} \mathrm{~mol}^{-1}$ for $\mathrm{X}=\mathrm{CH}_{2}(\mathbf{1 8}, \mathbf{1 9})$ and $12 \mathrm{~kJ} \mathrm{~mol}^{-1}$ for $\mathrm{X}=\mathrm{O}(\mathbf{2 0}, \mathbf{2 1})$, overestimated by rapport to the experimental values in solution but in the same order. Thus, it appears that $Z=M$ and $E=m$.

Respect to the cyclohexyl conformation of the derivative 22, it has been determined that the amide group occupies an equatorial position. It was confirmed by the detection of large coupling constant ${ }^{3} J$ between the axial H-1' $(\delta=3.82 \mathrm{ppm})$ and the neighboring axial H-2' $(\delta=1.63 \mathrm{ppm})$ in the ${ }^{1} \mathrm{H}$ NMR spectrum $\left({ }^{3} J_{1 \mathrm{ax}, 2 \mathrm{ax} x^{\prime}}=10.2 \mathrm{~Hz}\right)$. This is also the minimum energy conformation.

The comparison of experimental chemical shifts $(\delta, \mathrm{ppm})$ with calculated absolute shieldings $(\sigma, \mathrm{ppm})$ was performed to confirm the assignment of position and rotation isomers. The only atom that is sensitive and reliable is the ${ }^{13} \mathrm{C}$ of the $\mathrm{C}=\mathrm{O}$ group. This carbon is sensitive because for this signal a large difference $(\Delta \sigma \approx 5 \mathrm{ppm})$ between the $E$ and $Z$ isomers is observed and it is reliable because the other protons of Table 2 included those of the $\mathrm{NH}$ depend on the concentration and cannot be used. We have transformed the $\sigma^{13} \mathrm{C}$ into $\delta^{13} \mathrm{C}$ using the equation $\delta^{13} \mathrm{C}=175.7-0.963 \sigma^{13} \mathrm{C} .{ }^{28}$ With the $Z=M$ and $E=m$ assignment, we found $\delta^{13} \mathrm{Cexp}$. $=$ $(1.020 \pm 0.001) * \delta^{13}$ Ccalcd., $\mathrm{n}=8, \mathrm{R}^{2}=0.971$ (the opposite assignment yields and absurd relationship with an intercept of $330 \mathrm{ppm}$ !). Therefore, the assignment based on the energies has been confirmed by the chemical shifts. 


\section{Biological activity}

To explore the biological activity of the 1,2,3-triazoles presented here, competitive binding assays have been performed in membranes from HEK-293 EBNA cells expressed with human $\mathrm{CB}_{1}$ cannabinoid receptor. Therefore, the ability of 7a-11a, 13a-17a, 7b-10b, 14b, 15b, 10c, 1822 to bind this receptor has been evaluated by measuring the displacement of the radioligand $\left[{ }^{3} \mathrm{H}\right]-\mathrm{CP} 55940$ from $\mathrm{CB}_{1}$ receptor. None of the evaluated compounds showed significant affinity for $\mathrm{CB}_{1}$ cannabinoid receptor. They displaced $\left[{ }^{3} \mathrm{H}\right]-\mathrm{CP} 55940$ in less than $45 \%$ indicating a low affinity.

\section{Conclusions}

New di- and trisubstituted derivatives of 1,2,3-triazoles have been synthesized and evaluated as cannabinoid ligands. Although the 1,2,3-triazole ring has recently proved to be an interesting cannabinoid scaffold, ${ }^{12,13}$ none of the 1,2,3-triazoles evaluated here showed significant affinity for the $\mathrm{CB}_{1}$ cannabinoid receptor. Nevertheless, some interesting results concerning synthesis and reactivity of this ring system have been found. The diversity of chemical structures of the 1,2,3-triazole family and their useful biological activities make these compounds attractive targets in synthetic organic and medicinal chemistry.

\section{Experimental Section}

General. All starting materials were commercially available research grade chemicals and used without further purification. Dichloromethane was distilled over $\mathrm{CaCl}_{2}$. Silical gel $60 \mathrm{~F}_{254}$ (Merck) was used for TLC, and the spots were detected with UV light (254 nm). Flash column chromatography was carried out on silica gel 60 (Merck). Melting points were determined on a Reichert Jung Thermovar apparatus and are uncorrected. ${ }^{1} \mathrm{H}$ NMR spectra were recorded on a Varian Gemini $\left[200 \mathrm{MHz}\left({ }^{1} \mathrm{H}\right), 50 \mathrm{MHz}\left({ }^{13} \mathrm{C}\right)\right]$ spectrometer, Varian Inova 300 or $400[(300$ $\mathrm{MHz}\left({ }^{1} \mathrm{H}\right), 75 \mathrm{MHz}\left({ }^{13} \mathrm{C}\right)$ or $\left.400 \mathrm{MHz}\left({ }^{1} \mathrm{H}\right), 100 \mathrm{MHz}\left({ }^{13} \mathrm{C}\right)\right]$ spectrometer and Varian Unity 500 $\left[500 \mathrm{MHz}\left({ }^{1} \mathrm{H}\right), 125 \mathrm{MHz}\left({ }^{13} \mathrm{C}\right)\right]$ spectrometer with TMS as internal reference. Multiplicity were denoted by s (singlet), bs (broad singlet), d (doublet), t (triplet), q (quartet), quin (quintet), dd (double doublet) and $\mathrm{m}$ (multiplet). Mass spectra were determined on a MSD-Serie 1100 Hewlett Packard apparatus. Elemental analyses were performed with a Heraeus CHN-O Rapid analyser. Analyses indicated by the symbols of the elements or functions were within $\pm 0.4 \%$ of the theoretical values.

2,4-Dichloro-1-(4-methylphenyl)ethynylbenzene (1e). To a mixture of palladium diacetate (42.6 mg, $0.19 \mathrm{mmol}$ ), triphenylphosphine (99.7 mg, $0.38 \mathrm{mmol}), \mathrm{CH}_{3} \mathrm{CN}(8.6 \mathrm{~mL})$ and $\mathrm{H}_{2} \mathrm{O}(0.9$ $\mathrm{mL}$ ) in a Schlenk tube was added under nitrogen a well-stirred mixture of 1-ethynyl-4- 
methylbenzene $(0.24 \mathrm{~mL}, 1.9 \mathrm{mmol})$, 1,4-dichloro-2-iodobenzene $(0.51 \mathrm{~mL}, 3.8 \mathrm{mmol})$, triethylamine $(0.66 \mathrm{~mL}, 4.7 \mathrm{mmol})$, tetrabutylammonium hydrogen sulfate $(645.1 \mathrm{mg}, 1.9$ $\mathrm{mmol}), \mathrm{CH}_{3} \mathrm{CN}(8.6 \mathrm{~mL})$ and $\mathrm{H}_{2} \mathrm{O}(0.9 \mathrm{~mL})$. The reaction was stirred at room temperature for 3 h. The mixture was then hydrolyzed by $\mathrm{H}_{2} \mathrm{O}(15 \mathrm{~mL})$ and extracted with $\mathrm{Et}_{2} \mathrm{O}(3 \mathrm{X} 15 \mathrm{~mL})$. The combined organic extracts were dried over $\mathrm{MgSO}_{4}$. Evaporation of the solvent under reduced pressure afforded a solid, which was purified by column chromatography (cyclohexane) and recrystallized from cyclohexane to give the alkyne 1e. White solid (491.2 mg, 99\%); m.p. 69-71 ${ }^{\circ} \mathrm{C}$. ${ }^{1} \mathrm{H}$ NMR $\left(200 \mathrm{MHz}, \mathrm{CDCl}_{3}, 25{ }^{\circ} \mathrm{C}\right): \delta=7.57\left(\mathrm{~d},{ }^{4} J_{3,5}=2.6 \mathrm{~Hz}, 1 \mathrm{H}, \mathrm{H}-3\right), 7.51\left(\mathrm{~d},{ }^{3} J_{\mathrm{o}, \mathrm{m}}=\right.$ $\left.8.2 \mathrm{~Hz}, 2 \mathrm{H}, \mathrm{H}_{o}\right), 7.37\left(\mathrm{~d},{ }^{3} J_{5,6}=8.5 \mathrm{~Hz}, 1 \mathrm{H}, \mathrm{H}-6\right), 7.24\left(\mathrm{dd},{ }^{3} J_{5,6}=8.5 \mathrm{~Hz},{ }^{4} J_{3,5}=2.6 \mathrm{~Hz}, 1 \mathrm{H}\right.$, $\mathrm{H}-5), 7.21\left(\mathrm{~d},{ }^{3} J_{\mathrm{o}, \mathrm{m}}=8.2 \mathrm{~Hz}, 2 \mathrm{H}, \mathrm{H}_{m}\right), 2.42\left(\mathrm{~s}, 3 \mathrm{H}, \mathrm{CH}_{3}\right) \mathrm{ppm} ;{ }^{13} \mathrm{C} \mathrm{NMR}\left(50 \mathrm{MHz}, \mathrm{CDCl}_{3}, 25\right.$ $\left.{ }^{\circ} \mathrm{C}\right): \delta=139.1(\mathrm{C}-2), 134.0\left(\mathrm{C}_{p}\right), 132.4(\mathrm{C}-6), 132.1(\mathrm{C}-4), 131.6\left(\mathrm{C}_{o}\right), 130.1(\mathrm{C}-3), 129.0\left(\mathrm{C}_{m}\right)$, 128.9 (C-5), 124.7 (C-1), $119.2\left(\mathrm{C}_{\text {ipso }}\right), 95.9$ (C-1'), $84.3\left(\mathrm{C}-2^{\prime}\right), 21.4\left(\mathrm{CH}_{3}\right) \mathrm{ppm}$. ESI-MS: m/z $(\%)=264$ (21), $262(88), 260(100)\left[\mathrm{M}^{+}\right] ; 225(30)\left[\mathrm{M}^{+}-35\right] ; 189$ (75) $\left[\mathrm{M}^{+}-71\right]$. Anal. Calcd. for $\mathrm{C}_{15} \mathrm{H}_{10} \mathrm{Cl}_{2}$ : C, 68.99; H, 3.86. Found: C, 69.07; H, 4.12.

\section{Synthesis of triazoles 2-6. General procedure}

A mixture of tri- $n$-butyltin azide $(0.86 \mathrm{~mL}, 3.15 \mathrm{mmol})$ with the appropriate alkyne 1 ( $3 \mathrm{mmol})$ was heated at $150{ }^{\circ} \mathrm{C}$ for $70 \mathrm{~h}$ in a sealed glass bottle. The resulting solution was purified by column chromatography (cyclohexane/AcOEt, 5:1) and recrystallized from (cyclohexane/AcOEt) to give the desired triazoles.

4-(4-Methylphenyl)-1H(2H)-[1,2,3]triazole (2). Yellow solid (191.0 mg, 40\%); m.p. 139-141 ${ }^{\circ} \mathrm{C}$. ${ }^{1} \mathrm{H}$ NMR $\left(200 \mathrm{MHz}, \mathrm{DMSO}-d_{6}, 25^{\circ} \mathrm{C}\right): \delta=14.91$ (sw, $\left.1 \mathrm{H}, \mathrm{NH}\right), 8.08$ (s, $\left.1 \mathrm{H}, \mathrm{H}-5\right), 7.61(\mathrm{~d}$, $\left.{ }^{3} J_{\mathrm{o}, \mathrm{m}}=7.8 \mathrm{~Hz}, 2 \mathrm{H}, \mathrm{H}_{o}\right), 7.11\left(\mathrm{~d},{ }^{3} J_{\mathrm{o}, \mathrm{m}}=7.8 \mathrm{~Hz}, 2 \mathrm{H}, \mathrm{H}_{m}\right), 2.21\left(\mathrm{~s}, 3 \mathrm{H}, \mathrm{CH}_{3}\right) \mathrm{ppm} ;{ }^{13} \mathrm{C} \mathrm{NMR}(75$ MHz, DMSO- $\left.d_{6}, 25^{\circ} \mathrm{C}\right): \delta=147.4(\mathrm{C}-4), 137.7\left(\mathrm{C}_{p}\right), 129.3\left(\mathrm{C}_{m}, \mathrm{C}-5\right), 129.1\left(\mathrm{C}_{i p s o}\right), 125.4\left(\mathrm{C}_{o}\right)$, $20.8\left(\mathrm{CH}_{3}\right)$ ppm. ESI-MS: m/z $(\%)=182\left[\mathrm{M}^{+}+\mathrm{Na}\right] ; 160\left[\mathrm{M}^{+}+1\right]$. Anal. Calcd. for $\mathrm{C}_{9} \mathrm{H}_{9} \mathrm{~N}_{3}: \mathrm{C}$, 67.90; H, 5.70, N, 26.40. Found: C, 67.81; H, 5.75; N 26.49.

4-(4-Methoxyphenyl)-1H(2H)-[1,2,3]triazole (3). Yellow solid (157.7 mg, 30\%); m.p. 163-165 ${ }^{\circ} \mathrm{C}$. ${ }^{1} \mathrm{H}$ NMR $\left(200 \mathrm{MHz}, \mathrm{CDCl}_{3}, 25{ }^{\circ} \mathrm{C}\right): \delta=7.91(\mathrm{~s}, 1 \mathrm{H}, \mathrm{H}-5), 7.76\left(\mathrm{~d},{ }^{3} J_{\mathrm{o}, \mathrm{m}}=8.0 \mathrm{~Hz}, 2 \mathrm{H}, \mathrm{H}_{o}\right)$, $7.00\left(\mathrm{~d},{ }^{3} J_{\mathrm{o}, \mathrm{m}}=8.0 \mathrm{~Hz}, 2 \mathrm{H}, \mathrm{H}_{m}\right), 3.87\left(3 \mathrm{H}, \mathrm{s}, \mathrm{CH}_{3}\right) \mathrm{ppm} ;{ }^{13} \mathrm{C} \mathrm{NMR}\left(75 \mathrm{MHz}, \mathrm{CDCl}_{3}, 25{ }^{\circ} \mathrm{C}\right): \delta$ $=159.5\left(\mathrm{C}_{p}\right), 147.5(\mathrm{C}-4), 126.5(\mathrm{C}-5), 127.3\left(\mathrm{C}_{o}\right), 123.1\left(\mathrm{C}_{i p s o}\right), 114.7\left(\mathrm{C}_{m}\right), 55.5\left(\mathrm{CH}_{3}\right) \mathrm{ppm}$. ESI-MS: $\mathrm{m} / \mathrm{z}(\%)=198\left[\mathrm{M}^{+}+\mathrm{Na}\right] ; 177\left[\mathrm{M}^{+}+2\right] ; 176\left[\mathrm{M}^{+}+1\right]$. Anal. Calcd. for $\mathrm{C}_{9} \mathrm{H}_{9} \mathrm{~N}_{3} \mathrm{O}: \mathrm{C}$, 61.70; H, 5.18, N, 23.99. Found: C, 61.85; H, 5.15; N 24.09.

4-(4-Bromo-2-fluorophenyl)-1H(2H)-[1,2,3]triazole (4). Yellow solid (477.1 mg, 66\%); m.p. 139-141 ${ }^{\circ} \mathrm{C}$. ${ }^{1} \mathrm{H}$ NMR (200 MHz, DMSO- $\left.d_{6}, 25^{\circ} \mathrm{C}\right): \delta=11.33$ (sw, $\left.1 \mathrm{H}, \mathrm{NH}\right), 8.13$ (s, $\left.1 \mathrm{H}, \mathrm{H}-5\right)$, $7.87\left(\mathrm{dd},{ }^{3} J_{5^{\prime}, 6^{\prime}}=8.3 \mathrm{~Hz},{ }^{4} J_{6^{\prime}, \mathrm{F}}=8.1 \mathrm{~Hz}, 1 \mathrm{H}, \mathrm{H}-66^{\prime}\right), 7.57\left(\mathrm{dd},{ }^{3} J_{3^{\prime}, \mathrm{F}}=10.6 \mathrm{~Hz},{ }^{4} J_{3^{\prime}, 5^{\prime}}=2.0 \mathrm{~Hz}, 1 \mathrm{H}\right.$, H-3'), $7.41\left(\mathrm{ddd},{ }^{3} J_{5^{\prime}, 6^{\prime}}=8.3 \mathrm{~Hz},{ }^{4} J_{3^{\prime}, 5^{\prime}}=2.0 \mathrm{~Hz},{ }^{5} J_{5^{\prime}, \mathrm{F}}=0.6 \mathrm{~Hz}, 1 \mathrm{H}, \mathrm{H}-5{ }^{\prime}\right) \mathrm{ppm} ;{ }^{13} \mathrm{C} \mathrm{NMR}(75$ MHz, DMSO- $\left.d_{6}, 25^{\circ} \mathrm{C}\right): \delta=158.9$ (d, $\left.{ }^{1} J_{2^{\prime}, \mathrm{F}}=252.5 \mathrm{~Hz}, \mathrm{C}-2^{\prime}\right), 132.3(\mathrm{C}-4), 129.6(\mathrm{C}-5), 128.6$ (C-6'), 128.5 (C-5'), 121.5 (C-4'), 119.8 (d, $\left.{ }^{2} J_{3^{\prime}, \mathrm{F}}=38.8 \mathrm{~Hz}, \mathrm{C}-3^{\prime}\right), 118.1$ (C-1') ppm. ESI-MS: $\mathrm{m} / \mathrm{z}(\%)=244,242\left[\mathrm{M}^{+}+1\right]$. Anal. Calcd. for $\mathrm{C}_{8} \mathrm{H}_{5} \mathrm{BrFN}_{3}$ : C, 39.70; H, 2.08, N, 17.36. Found: C, $39.82 ; \mathrm{H}, 2.12 ; \mathrm{N} 17.15$. 
4-(4-Chlorophenyl)-5-phenyl-1H(2H)-[1,2,3]triazole (5). White solid (413.2 mg, 54\%); m.p. 124-126 ${ }^{\circ} \mathrm{C}$. ${ }^{1} \mathrm{H}$ NMR $\left(200 \mathrm{MHz}, \mathrm{CDCl}_{3}, 25^{\circ} \mathrm{C}\right): \delta=7.51-7.46\left(\mathrm{~m}, 4 \mathrm{H}, \mathrm{H}_{o}, \mathrm{H}_{o}\right)$ ) 7.38-7.35 (m, $\left.3 \mathrm{H}, \mathrm{H}_{m}, \mathrm{H}_{p}\right), 7.31\left(\mathrm{dt},{ }^{3} J_{\mathrm{o}, \mathrm{m}}=8.6 \mathrm{~Hz},{ }^{4} J_{\mathrm{m}, \mathrm{m}}={ }^{5} J_{\mathrm{o}, \mathrm{m}}=2.2 \mathrm{~Hz}, 2 \mathrm{H}, \mathrm{H}_{m}\right) \mathrm{ppm} ;{ }^{13} \mathrm{C} \mathrm{NMR}(50.5$ $\left.\mathrm{MHz}, \mathrm{CDCl}_{3}, 25^{\circ} \mathrm{C}\right): \delta=142.4(\mathrm{C}-5), 142.0(\mathrm{C}-4), 134.6\left(\mathrm{C}_{p}\right), 129.6\left(\mathrm{C}_{\text {ipso }}\right), 129.4\left(\mathrm{C}_{o}\right), 128.9$ $\left(\mathrm{C}_{m}, \mathrm{C}_{p^{\prime}}\right), 128.8\left(\mathrm{C}_{m^{\prime}}\right), 128.6\left(\mathrm{C}_{\text {ipso }}\right), 128.2\left(\mathrm{C}_{o^{\prime}}\right)$ ppm. ESI-MS: m/z $(\%)=278\left[\mathrm{M}^{+}+\mathrm{Na}\right], 256$ $\left[\mathrm{M}^{+}\right.$]. Anal. Calcd. for $\mathrm{C}_{14} \mathrm{H}_{10} \mathrm{ClN}_{3}$ : C, 67.76; H, 3.94, N, 16.43. Found: C, 67.93; H, 3.84; N 16.36 .

4-(2,4-Dichlorophenyl)-5-(4-methylphenyl)-1H(2H)-[1,2,3]triazole (6). White solid (611.1 mg, 67\%); m.p. $54-56{ }^{\circ} \mathrm{C} .{ }^{1} \mathrm{H}$ NMR $\left(200 \mathrm{MHz}, \mathrm{CDCl}_{3}, 25^{\circ} \mathrm{C}\right): \delta=13.56$ (sw, $\left.1 \mathrm{H}, \mathrm{NH}\right), 7.45$ (d, $\left.{ }^{4} J_{3^{\prime}, 5^{\prime}}=2.2 \mathrm{~Hz}, 1 \mathrm{H}, \mathrm{H}-3^{\prime}\right), 7.39$ (d, $\left.{ }^{3} J_{5^{\prime}, 6^{\prime}}=8.3 \mathrm{~Hz}, 1 \mathrm{H}, \mathrm{H}-6^{\prime}\right), 7.36-7.33$ (m, $3 \mathrm{H}, \mathrm{H}-5^{\prime}, \mathrm{H}_{o}$ ), 7.12 $\left(\mathrm{d},{ }^{3} \mathrm{~J}_{\mathrm{o}, \mathrm{m}}=7.8 \mathrm{~Hz}, 2 \mathrm{H}, \mathrm{H}_{m}\right), 2.26\left(\mathrm{~s}, 3 \mathrm{H}, \mathrm{CH}_{3}\right) \mathrm{ppm} ;{ }^{13} \mathrm{C} \mathrm{NMR}\left(75 \mathrm{MHz}, \mathrm{CDCl}_{3}, 25{ }^{\circ} \mathrm{C}\right): \delta=$ 144.2 (C-5), 140.4 (C-4), 138.7 (C C $_{p}$, 132.7 (C-2'), 132.6 (C-4'), 131.8 (C-3'), 131.7 (C-1'), 131.1 (C-6'), $130.3\left(\mathrm{C}^{-5}\right), 129.5\left(\mathrm{C}_{m}\right), 126.8\left(\mathrm{C}_{o}\right), 126.3\left(\mathrm{C}_{\text {ipso }}\right), 21.4\left(\mathrm{CH}_{3}\right) \mathrm{ppm}$. ESI-MS: m/z $(\%)=$

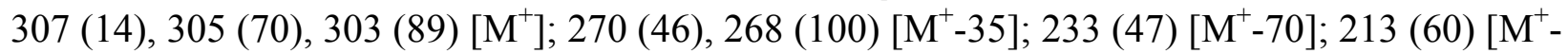
90]. Anal. Calcd. for $\mathrm{C}_{15} \mathrm{H}_{11} \mathrm{Cl}_{2} \mathrm{~N}_{3}$ : C, 59.23; H, 3.65; N, 13.81. Found: $\mathrm{C}, 59.65 ; \mathrm{H}, 3.85 ; \mathrm{N}$, 13.09 .

\section{Preparation of triazoles 7-17 by $\boldsymbol{N}$-alkylation of 2-6. General procedure}

To a solution of 1,2,3-triazole 2-6 $(0.5 \mathrm{mmol})$ in acetonitrile $(3 \mathrm{~mL})$ was added $\mathrm{K}_{2} \mathrm{CO}_{3}(83.0 \mathrm{mg}$, $0.6 \mathrm{mmol}), \mathrm{KOH}(84.0 \mathrm{mg}, 1.5 \mathrm{mmol})$ and $\mathrm{Bu}_{4} \mathrm{NBr}(4.0 \mathrm{mg}, 0.013 \mathrm{mmol})$. The mixture was stirred for $5 \mathrm{~min}$. at r.t. Then the appropriate alkyl bromide $(0.6 \mathrm{mmol})$ was added and the mixture was stirred at reflux for $5 \mathrm{~h}$ for 7-12, 16-17 and for $2 \mathrm{~h}$ for 13-15. The resulting solution was filtered and the remaining solid material was washed with $\mathrm{Et}_{2} \mathrm{O}(20 \mathrm{~mL})$. Evaporation of the solvent afforded an oil residue. From the oily crude the different regioisomers were separated by column chromatography eluting with cyclohexane/AcOEt $(20: 1)$ to get the isomers 7a-15a then eluting with cyclohexane/ AcOEt (10:1) to separate the isomers 7b-15b and 7c-15c. The regioisomers 16-17 were separated by column chromatography eluting with cyclohexane/AcOEt $(10: 1)$ to get the isomers 16a-17a then eluting with cyclohexane/AcOEt $(5: 1)$ to obtain the isomer 16b.

2-Pentyl-4-(4-methylphenyl)-2H-[1,2,3]triazole (7a). Colourless oil (71.1 mg, 62\%). ${ }^{1} \mathrm{H} \mathrm{NMR}$ $\left(200 \mathrm{MHz}, \mathrm{CDCl}_{3}, 25^{\circ} \mathrm{C}\right): \delta=7.69(\mathrm{~s}, 1 \mathrm{H}, \mathrm{H}-5), 7.59\left(\mathrm{~d},{ }^{3} J_{\mathrm{o}, \mathrm{m}}=8.0 \mathrm{~Hz}, 2 \mathrm{H}, \mathrm{H}_{o}\right), 7.18\left(\mathrm{~d},{ }^{3} J_{\mathrm{o}, \mathrm{m}}\right.$ $\left.=8.0 \mathrm{~Hz}, 2 \mathrm{H}, \mathrm{H}_{m}\right), 4.35\left(\mathrm{t},{ }^{3} J_{1^{\prime}, 2^{\prime}}=7.0 \mathrm{~Hz}, 2 \mathrm{H}, \mathrm{H}-1^{\prime}\right), 2.29\left(\mathrm{~s}, 3 \mathrm{H}, \mathrm{CH}_{3}\right), 1.91$ (quin, ${ }^{3} J_{1^{\prime}, 2^{\prime}}={ }^{3} J_{2^{\prime}, 3^{\prime}}$ $\left.=7.0 \mathrm{~Hz}, 2 \mathrm{H}, \mathrm{H}-2^{\prime}\right), 1.29-1.21$ (m, $4 \mathrm{H}, \mathrm{H}-3$ ', H-4'), 0.82 (t, $\left.{ }^{3} J_{4^{\prime}, 5^{\prime}}=7.0 \mathrm{~Hz}, 3 \mathrm{H}, \mathrm{H}-5^{\prime}\right) \mathrm{ppm} ;{ }^{13} \mathrm{C}$ NMR (75 MHz, $\left.\mathrm{CDCl}_{3}, 25^{\circ} \mathrm{C}\right): \delta=147.4(\mathrm{C}-4), 138.0\left(\mathrm{C}_{p}\right), 130.0(\mathrm{C}-5), 129.4\left(\mathrm{C}_{m}\right), 127.6$ $\left(\mathrm{C}_{i p s o}\right), 125.7\left(\mathrm{C}_{o}\right), 55.0$ (C-1'), 29.5 (C-2'), $28.6\left(\mathrm{C}^{\prime} 3^{\prime}\right), 22.1\left(\mathrm{C}-4{ }^{\prime}\right), 21.2\left(\mathrm{CH}_{3}\right), 13.8\left(\mathrm{C}-5^{\prime}\right) \mathrm{ppm}$. ESI-MS: $\mathrm{m} / \mathrm{z}(\%)=229(100)\left[\mathrm{M}^{+}\right] ; 186$ (73) $\left[\mathrm{M}^{+}-43\right] ; 131$ (52) $\left[\mathrm{M}^{+}-98\right] ; 118$ (51) $\left[\mathrm{M}^{+}-111\right]$; $116(59)\left[\mathrm{M}^{+}\right.$-113]. Anal. Calcd. for $\mathrm{C}_{14} \mathrm{H}_{19} \mathrm{~N}_{3}$ : C, 73.33; H, 8.35; N, 18.32. Found: C, 73.28; H, $8.53 ; \mathrm{N} 18.13$.

1-Pentyl-4-(4-methylphenyl)-1H-[1,2,3]triazole (7b). White solid (32.6 mg, 28\%); m.p. 73-75 ${ }^{\circ} \mathrm{C}$ (cyclohexane/AcOEt). ${ }^{1} \mathrm{H} \mathrm{NMR}\left(200 \mathrm{MHz}, \mathrm{CDCl}_{3}, 25{ }^{\circ} \mathrm{C}\right): \delta=7.64\left(\mathrm{~d},{ }^{3} J_{\mathrm{o}, \mathrm{m}}=8.2 \mathrm{~Hz}, 2 \mathrm{H}\right.$, 
$\left.\mathrm{H}_{o}\right), 7.62(\mathrm{~s}, 1 \mathrm{H}, \mathrm{H}-5), 7.14\left(\mathrm{~d},{ }^{3} J_{\mathrm{o}, \mathrm{m}}=8.2 \mathrm{~Hz}, 2 \mathrm{H}, \mathrm{H}_{m}\right), 4.28\left(\mathrm{t},{ }^{3} J_{1^{\prime}, 2^{\prime}}=7.0 \mathrm{~Hz}, 2 \mathrm{H}, \mathrm{H}-1^{\prime}\right), 2.29$ (s, $3 \mathrm{H}, \mathrm{CH}_{3}$ ), 1.85 (quin, ${ }^{3} J_{1^{\prime}, 2^{\prime}}={ }^{3} J_{2^{\prime}, 3^{\prime}}=7.0 \mathrm{~Hz}, 2 \mathrm{H}, \mathrm{H}-2^{\prime}$ ), 1.29-1.18 (m, $\left.4 \mathrm{H}, \mathrm{H}-3^{\prime}, \mathrm{H}-4^{\prime}\right), 0.82$ (t, $\left.{ }^{3} J_{4^{\prime}, 5^{\prime}}=7.0 \mathrm{~Hz}, 3 \mathrm{H}, \mathrm{H}-5^{\prime}\right) \mathrm{ppm} ;{ }^{13} \mathrm{C}$ NMR $\left(75 \mathrm{MHz}, \mathrm{CDCl}_{3}, 25{ }^{\circ} \mathrm{C}\right): \delta=147.7$ (C-4), 137.8 $\left(\mathrm{C}_{p}\right), 129.4\left(\mathrm{C}_{m}\right), 127.8\left(\mathrm{C}_{\text {ipso }}\right), 125.5\left(\mathrm{C}_{o}\right), 119.0(\mathrm{C}-5), 50.3$ (C-1'), $30.0\left(\mathrm{C}-2^{\prime}\right), 28.5\left(\mathrm{C}-3^{\prime}\right), 22.0$ (C-4'), $21.2\left(\mathrm{CH}_{3}\right), 13.8\left(\mathrm{C}-5^{\prime}\right)$ ppm. ESI-MS: m/z (\%) = $229(50)\left[\mathrm{M}^{+}\right] ; 159(43)\left[\mathrm{M}^{+}-70\right] ; 131$ (100) $\left[\mathrm{M}^{+}\right.$-98]. Anal. Calcd. for $\mathrm{C}_{14} \mathrm{H}_{19} \mathrm{~N}_{3}$ : $\mathrm{C}, 73.33 ; \mathrm{H}, 8.35 ; \mathrm{N}, 18.32$. Found: $\mathrm{C}, 73.48 ; \mathrm{H}, 8.47$; N 18.01 .

1-Pentyl-5-(4-methylphenyl)-1H-[1,2,3]triazole (7c). White solid (5.7 mg, 5\%); m.p. 102-104 ${ }^{\circ} \mathrm{C}$ (cyclohexane/AcOEt). ${ }^{1} \mathrm{H}$ NMR $\left(500 \mathrm{MHz}, \mathrm{CDCl}_{3}, 25^{\circ} \mathrm{C}\right): \delta=7.56(\mathrm{~s}, 1 \mathrm{H}, \mathrm{H}-4), 7.19-7.16$ $\left(\mathrm{m}, 4 \mathrm{H}, \mathrm{H}_{o}, \mathrm{H}_{m}\right), 4.26\left(\mathrm{t},{ }^{3} J_{1^{\prime}, 2^{\prime}}=7.0 \mathrm{~Hz}, 2 \mathrm{H}, \mathrm{H}-1^{\prime}\right), 2.33\left(\mathrm{~s}, 3 \mathrm{H}, \mathrm{CH}_{3}\right.$ ), 1.73 (quin, ${ }^{3} J_{1^{\prime}, 2^{\prime}}={ }^{3} J_{2^{\prime}, 3^{\prime}}=$ $\left.7.0 \mathrm{~Hz}, 2 \mathrm{H}, \mathrm{H}-2^{\prime}\right), 1.27-1.15$ (m, $\left.4 \mathrm{H}, \mathrm{H}-3^{\prime}, \mathrm{H}-4^{\prime}\right), 0.74$ (t, ${ }^{3} J_{4^{\prime}, 5^{\prime}}=7.0 \mathrm{~Hz}, 3 \mathrm{H}, \mathrm{H}-5$ ') ppm; ${ }^{13} \mathrm{C}$ $\operatorname{NMR}\left(75 \mathrm{MHz}, \mathrm{CDCl}_{3}, 25^{\circ} \mathrm{C}\right): \delta=139.5\left(\mathrm{C}_{p}\right), 137.7(\mathrm{C}-5), 132.9(\mathrm{C}-4), 129.7\left(\mathrm{C}_{m}\right), 128.6\left(\mathrm{C}_{o}\right)$, $124.3\left(\mathrm{C}_{\text {ipso }}\right), 48.2\left(\mathrm{C}-1^{\prime}\right), 29.8$ (C-2'), 28.5 (C-3'), $22.0\left(\mathrm{C}^{\prime} 4^{\prime}\right), 21.3\left(\mathrm{CH}_{3}\right), 13.8$ (C-5') ppm. ESIMS: $\mathrm{m} / \mathrm{z}(\%)=229(16)\left[\mathrm{M}^{+}\right] ; 131(100)\left[\mathrm{M}^{+}-98\right] ; 116(67)\left[\mathrm{M}^{+}-113\right]$. Anal. Calcd. for $\mathrm{C}_{14} \mathrm{H}_{19} \mathrm{~N}_{3}: \mathrm{C}, 73.33 ; \mathrm{H}, 8.35 ; \mathrm{N}, 18.32$. Found: C, 73.74; H, 8.25; N 18.01 .

4-(4-Methoxyphenyl)-2-pentyl-2H-[1,2,3]triazole (8a). Yellow solid (55.2 mg, 45\%); m.p. 52-54 ${ }^{\circ} \mathrm{C}$ (cyclohexane/AcOEt). ${ }^{1} \mathrm{H}$ NMR $\left(200 \mathrm{MHz}, \mathrm{CDCl}_{3}, 25^{\circ} \mathrm{C}\right): \delta=7.75(\mathrm{~s}, 1 \mathrm{H}, \mathrm{H}-5), 7.71$ $\left(\mathrm{d},{ }^{3} J_{\mathrm{o}, \mathrm{m}}=9.0 \mathrm{~Hz}, 2 \mathrm{H}, \mathrm{H}_{o}\right), 6.96\left(\mathrm{~d},{ }^{3} J_{\mathrm{o}, \mathrm{m}}=9.0 \mathrm{~Hz}, 2 \mathrm{H}, \mathrm{H}_{m}\right), 4.43\left(\mathrm{t},{ }^{3} J_{1^{\prime}, 2^{\prime}}=7.0 \mathrm{~Hz}, 2 \mathrm{H}, \mathrm{H}-1^{\prime}\right)$, 3.85 (s, $3 \mathrm{H}, \mathrm{CH}_{3}$ ), 2.00 (quin, ${ }^{3} J_{1^{\prime}, 2^{\prime}}={ }^{3} J_{2^{\prime}, 3^{\prime}}=7.0 \mathrm{~Hz}, 2 \mathrm{H}, \mathrm{H}-2^{\prime}$ ), 1.38-1.26 (m, $4 \mathrm{H}, \mathrm{H}-3{ }^{\prime}, \mathrm{H}-4^{\prime}$ ), $0.91\left(\mathrm{t},{ }^{3} J_{4^{\prime}, 5^{\prime}}=7.0 \mathrm{~Hz}, 3 \mathrm{H}, \mathrm{H}-5^{\prime}\right) \mathrm{ppm} ;{ }^{13} \mathrm{C} \mathrm{NMR}\left(50.5 \mathrm{MHz}, \mathrm{CDCl}_{3}, 25{ }^{\circ} \mathrm{C}\right): \delta=159.7\left(\mathrm{C}_{p}\right)$, 147.3 (C-4), $130.0(\mathrm{C}-5), 127.1\left(\mathrm{C}_{o}\right), 123.3\left(\mathrm{C}_{i p s o}\right), 114.2\left(\mathrm{C}_{m}\right), 55.3\left(\mathrm{CH}_{3}\right), 55.0\left(\mathrm{C}-1{ }^{\prime}\right), 29.5(\mathrm{C}-$ 2'), 28.6 (C-3'), 22.1 (C-4'), 13.9 (C-5') ppm. ESI-MS: m/z (\%) = $245(100)\left[\mathrm{M}^{+}\right] ; 202(52)\left[\mathrm{M}^{+}-\right.$ 43]; 133 (54) $\left[\mathrm{M}^{+}-112\right] ; 132(88)\left[\mathrm{M}^{+}-113\right]$. Anal. Calcd. for $\mathrm{C}_{14} \mathrm{H}_{19} \mathrm{~N}_{3} \mathrm{O}: \mathrm{C}, 68.54 ; \mathrm{H}, 7.81 ; \mathrm{N}$, 17.13. Found: C, 68.65; H, 8.12; N 16.97.

4-(4-Methoxyphenyl)-1-pentyl-1H-[1,2,3]triazole (8b). Yellow solid (30.7 mg, 25\%); m.p. 100-102 ${ }^{\circ} \mathrm{C}$ (cyclohexane/AcOEt). ${ }^{1} \mathrm{H}$ NMR $\left(200 \mathrm{MHz}, \mathrm{CDCl}_{3}, 25{ }^{\circ} \mathrm{C}\right): \delta=7.76\left(\mathrm{~d},{ }^{3} J_{\mathrm{o}, \mathrm{m}}=8.7\right.$ $\left.\mathrm{Hz}, 2 \mathrm{H}, \mathrm{H}_{o}\right), 7.67(\mathrm{~s}, 1 \mathrm{H}, \mathrm{H}-5), 6.96\left(\mathrm{~d},{ }^{3} J_{\mathrm{o}, \mathrm{m}}=8.7 \mathrm{~Hz}, 2 \mathrm{H}, \mathrm{H}_{m}\right), 4.38\left(\mathrm{t},{ }^{3} J_{1^{\prime}, 2^{\prime}}=7.2 \mathrm{~Hz}, 2 \mathrm{H}, \mathrm{H}-\right.$ 1'), 3.85 (s, $3 \mathrm{H}, \mathrm{CH}_{3}$ ), 1.95 (quin, ${ }^{3} J_{1^{\prime}, 2^{\prime}}={ }^{3} J_{2^{\prime}, 3^{\prime}}=7.2 \mathrm{~Hz}, 2 \mathrm{H}, \mathrm{H}-2^{\prime}$ ), 1.39-1.33 (m, $4 \mathrm{H}, \mathrm{H}-3^{\prime}, \mathrm{H}-$ $\left.4^{\prime}\right), 0.91\left(\mathrm{t},{ }^{3} J_{4^{\prime}, 5^{\prime}}=6.7 \mathrm{~Hz}, 3 \mathrm{H}, \mathrm{H}-5^{\prime}\right) \mathrm{ppm} ;{ }^{13} \mathrm{C} \mathrm{NMR}\left(50.5 \mathrm{MHz}, \mathrm{CDCl}_{3}, 25^{\circ} \mathrm{C}\right): \delta=159.5\left(\mathrm{C}_{p}\right)$, $\left.147.5(\mathrm{C}-4), 126.9\left(\mathrm{C}_{o}\right), 123.5\left(\mathrm{C}_{\text {ipso }}\right), 118.6(\mathrm{C}-5), 114.2\left(\mathrm{C}_{m}\right), 56.3\left(\mathrm{CH}_{3}\right), 50.0(\mathrm{C}-1)\right), 30.0(\mathrm{C}-$ 2'), 28.6 (C-3'), 22.1 (C-4'), 13.8 (C-5') ppm. ESI-MS: m/z (\%) = 245 (76) $\left[\mathrm{M}^{+}\right] ; 175$ (93) [M+70]; 147 (100) [ $\left.\mathrm{M}^{+}-98\right] ; 132$ (79) $\left[\mathrm{M}^{+}-113\right]$. Anal. Calcd. for $\mathrm{C}_{14} \mathrm{H}_{19} \mathrm{~N}_{3} \mathrm{O}: \mathrm{C}, 68.54 ; \mathrm{H}, 7.81 ; \mathrm{N}$, 17.13. Found: C, 68.42; H, 7.97; N 17.40.

4-(4-Bromo-2-fluorophenyl)-2-pentyl-2H-[1,2,3]triazole (9a). Colourless oil (107.7 mg, 69\%). ${ }^{1} \mathrm{H}$ NMR $\left(200 \mathrm{MHz}, \mathrm{CDCl}_{3}, 25^{\circ} \mathrm{C}\right): \delta=7.84\left(\mathrm{~d},{ }^{5} J_{5, \mathrm{~F}}=3.8 \mathrm{~Hz}, 1 \mathrm{H}, \mathrm{H}-5\right), 7.80\left(\mathrm{dd},{ }^{3} J_{5^{\prime \prime}, 6}=8.5\right.$ $\left.\mathrm{Hz},{ }^{4} J_{6^{\prime \prime}, \mathrm{F}}=8.0 \mathrm{~Hz}, 1 \mathrm{H}, \mathrm{H}-6 "\right), 7.29-7.18$ (m, $\left.2 \mathrm{H}, \mathrm{H}-3 ", \mathrm{H}-5 "\right), 4.37\left(\mathrm{t},{ }^{3} J_{1^{\prime}, 2^{\prime}}=7.0 \mathrm{~Hz}, 2 \mathrm{H}, \mathrm{H}-1^{\prime}\right)$, 1.92 (quin, $\left.{ }^{3} J_{1^{\prime}, 2^{\prime}}={ }^{3} J_{2^{\prime}, 3^{\prime}}=7.0 \mathrm{~Hz}, 2 \mathrm{H}, \mathrm{H}-2^{\prime}\right), 1.34-1.31$ (m, $\left.4 \mathrm{H}, \mathrm{H}-3^{\prime}, \mathrm{H}-4^{\prime}\right), 0.82\left(\mathrm{t},{ }^{3} J_{4^{\prime}, 5}{ }^{\prime}=6.7\right.$ $\left.\mathrm{Hz}, 3 \mathrm{H}, \mathrm{H}-5^{\prime}\right) \mathrm{ppm} ;{ }^{13} \mathrm{C} \mathrm{NMR}\left(50.5 \mathrm{MHz}, \mathrm{CDCl}_{3}, 25^{\circ} \mathrm{C}\right): \delta=159.4\left(\mathrm{~d},{ }^{1} J_{2 ", \mathrm{~F}}=254.7 \mathrm{~Hz}, \mathrm{C}-2^{\prime \prime}\right)$, 140.9 (C-4), 133.4 (d, $\left.{ }^{4} J_{5, \mathrm{~F}}=12.5 \mathrm{~Hz}, \mathrm{C}-5\right), 129.1\left(\mathrm{~d},{ }^{3} J_{6^{\prime \prime}, \mathrm{F}}=4.4 \mathrm{~Hz}, \mathrm{C}-6 "\right), 127.8\left(\mathrm{~d},{ }^{4} J_{5^{\prime \prime}, \mathrm{F}}=3.5\right.$ $\mathrm{Hz}, \mathrm{C}-5 "), 121.8\left(\mathrm{~d},{ }^{3} J_{4 ", \mathrm{~F}}=9.9 \mathrm{~Hz}, \mathrm{C}-4 "\right), 119.6\left(\mathrm{~d},{ }^{2} J_{3^{\prime \prime}, \mathrm{F}}=25.1 \mathrm{~Hz}, \mathrm{C}-3 "\right), 117.7\left(\mathrm{~d},{ }^{2} J_{1^{\prime \prime}, \mathrm{F}}=12.6\right.$ 
Hz, C-1"), 55.1 (C-1'), 29.4 (C-2'), 28.6 (C-3'), 22.1 (C-4'), 13.9 (C-5') ppm. ESI-MS: m/z (\%) =

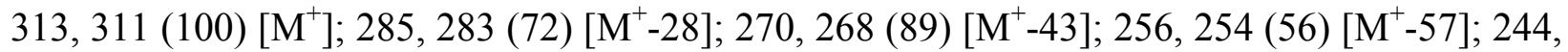

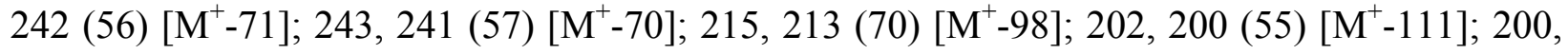
198 (49) $\left[\mathrm{M}^{+}-113\right]$. Anal. Calcd. for $\mathrm{C}_{13} \mathrm{H}_{15} \mathrm{BrFN}_{3}$ : C, 50.02; H, 4.84; N, 13.46. Found: C, 50.28; $\mathrm{H}, 4.48$; N 13.73 .

4-(4-Bromo-2-fluorophenyl)-1-pentyl-1H-[1,2,3]triazole (9b). Yellow solid (45.3 mg, 29\%); m.p. $71-73{ }^{\circ} \mathrm{C}$ (cyclohexane/AcOEt). ${ }^{1} \mathrm{H}$ NMR $\left(200 \mathrm{MHz}, \mathrm{CDCl}_{3}, 25{ }^{\circ} \mathrm{C}\right): \delta=8.12\left(\mathrm{t},{ }^{3} J_{5^{\prime \prime}, 6^{\prime \prime}}=\right.$ $\left.{ }^{4} J_{6, ", F}=8.2 \mathrm{~Hz}, 1 \mathrm{H}, \mathrm{H}-6 "\right), 7.82$ (d, $\left.{ }^{5} J_{5-\mathrm{F}}=3.8 \mathrm{~Hz}, 1 \mathrm{H}, \mathrm{H}-5\right), 7.35-7.15$ (m, $2 \mathrm{H}, \mathrm{H}-3$ ", H-5"), $4.33\left(\mathrm{t},{ }^{3} J_{1^{\prime}, 2^{\prime}}=7.0 \mathrm{~Hz}, 2 \mathrm{H}, \mathrm{H}-1^{\prime}\right), 1.89$ (quin, $\left.{ }^{3} J_{1^{\prime}, 2^{\prime}}={ }^{3} J_{2^{\prime}, 3^{\prime}}=7.0 \mathrm{~Hz}, 2 \mathrm{H}, \mathrm{H}-2^{\prime}\right), 1.33-1.26$ (m, 4 H, H-3', H-4'), 0.84 (t, $\left.{ }^{3} J_{4^{\prime}, 5^{\prime}}=6.9 \mathrm{~Hz}, 3 \mathrm{H}, \mathrm{H}-5^{\prime}\right) \mathrm{ppm} ;{ }^{13} \mathrm{C} \mathrm{NMR}\left(50.5 \mathrm{MHz}, \mathrm{CDCl}_{3}, 25{ }^{\circ} \mathrm{C}\right): \delta=$ $158.7\left(\mathrm{~d},{ }^{1} J_{2 ", \mathrm{~F}}=258.7 \mathrm{~Hz}, \mathrm{C}-2^{\prime \prime}\right), 140.2\left(\mathrm{~d},{ }^{3} J_{4, \mathrm{~F}}=3.1 \mathrm{~Hz}, \mathrm{C}-4\right), 128.7$ (d, $\left.{ }^{3} J_{6 ", F}=6.1 \mathrm{~Hz}, \mathrm{C}-6 "\right)$, $128.0\left(\mathrm{~d},{ }^{4} J_{5^{\prime \prime}, \mathrm{F}}=3.9 \mathrm{~Hz}, \mathrm{C}-5 "\right), 122.5\left(\mathrm{~d},{ }^{4} J_{5, \mathrm{~F}}=13.9 \mathrm{~Hz}, \mathrm{C}-5\right), 121.4$ (d, $\left.{ }^{3} J_{4 ", \mathrm{~F}}=8.9 \mathrm{~Hz}, \mathrm{C}-4 "\right)$, $119.2\left(\mathrm{~d},{ }^{2} J_{3 ", \mathrm{~F}}=25.1 \mathrm{~Hz}, \mathrm{C}-3 "\right), 117.9$ (d, $\left.{ }^{2} J_{1 ", \mathrm{~F}}=13.0 \mathrm{~Hz}, \mathrm{C}-1^{\prime \prime}\right), 50.4$ (C-1'), 29.9 (C-2'), 28.5

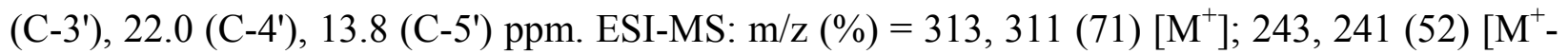
70]; 215, 213 (100) [ $\left.\mathrm{M}^{+}-98\right] ; 202,200$ (66) [ $\left.\mathrm{M}^{+}-111\right]$. Anal. Calcd. for $\mathrm{C}_{13} \mathrm{H}_{15} \mathrm{BrFN}_{3}$ : C, 50.02; H, 4.84; N, 13.46. Found: C, 50.19; H, 4.96; N 13.17.

4-(4-Chlorophenyl)-2-pentyl-5-phenyl-2H-[1,2,3]triazole (10a). Colorless oil (81.5 mg, 50\%). ${ }^{1} \mathrm{H}$ NMR $\left(200 \mathrm{MHz}, \mathrm{CDCl}_{3}, 25^{\circ} \mathrm{C}\right): \delta=7.46-7.37\left(\mathrm{~m}, 4 \mathrm{H}, \mathrm{H}_{o}, \mathrm{H}_{o}\right), 7.30-7.20\left(\mathrm{~m}, 5 \mathrm{H}, \mathrm{H}_{m}, \mathrm{H}_{p^{\prime}}\right.$, $\mathrm{H}_{m}$ ), $4.36\left(\mathrm{t},{ }^{3} J_{1^{\prime}, 2^{\prime}}=7.0 \mathrm{~Hz}, 2 \mathrm{H}, \mathrm{H}-1^{\prime}\right.$ ), 1.95 (quin, ${ }^{3} J_{1^{\prime}, 2^{\prime}}={ }^{3} J_{2^{\prime}, 3^{\prime}}=7.0 \mathrm{~Hz}, 2 \mathrm{H}, \mathrm{H}-2^{\prime}$ ), 1.32-1.25 (m, 4 H, H-3', H-4'), 0.83 (t, $\left.{ }^{3} J_{4}, 5^{\prime}=7.0 \mathrm{~Hz}, 3 \mathrm{H}, \mathrm{H}-5^{\prime}\right)$ ppm; ${ }^{13} \mathrm{C} \mathrm{NMR}\left(75 \mathrm{MHz}, \mathrm{CDCl}_{3}, 25{ }^{\circ} \mathrm{C}\right)$ : $\delta=144.2(\mathrm{C}-5), 142.9(\mathrm{C}-4), 134.1\left(\mathrm{C}_{p}\right), 130.9\left(\mathrm{C}_{i p s o}\right), 130.0\left(\mathrm{C}_{i p s o}\right), 129.4\left(\mathrm{C}_{o}\right), 128.7\left(\mathrm{C}_{m}\right)$, $128.6\left(\mathrm{C}_{m^{\prime}}\right), 128.3\left(\mathrm{C}_{p^{\prime}}\right), 128.2\left(\mathrm{C}_{o^{\prime}}\right), 55.6\left(\mathrm{C}-1^{\prime}\right), 29.9\left(\mathrm{C}-2^{\prime}\right), 29.1\left(\mathrm{C}-3^{\prime}\right), 22.6\left(\mathrm{C}-4^{\prime}\right), 14.6\left(\mathrm{C}-5^{\prime}\right)$ ppm. ESI-MS: m/z (\%) = 327 (42), 325 (100) $\left[\mathrm{M}^{+}\right] ; 296(58)\left[\mathrm{M}^{+}-29\right] ; 282$ (51) $\left[\mathrm{M}^{+}-43\right] ; 227$ (60) $\left[\mathrm{M}^{+}-98\right] ; 165$ (56) $\left[\mathrm{M}^{+}-160\right]$. Anal. Calcd. for $\mathrm{C}_{19} \mathrm{H}_{20} \mathrm{ClN}_{3}$ : C, 70.04; H, 6.19; N, 12.90 . Found: C, 70.44; H, 6.10; N 12.76.

4-(4-Chlorophenyl)-1-pentyl-5-phenyl-1H-[1,2,3]triazole (10b). White solid (50.5 mg, 31\%); m.p. 85-87 ${ }^{\circ} \mathrm{C}$ (cyclohexane/AcOEt). ${ }^{1} \mathrm{H}$ NMR $\left(200 \mathrm{MHz}, \mathrm{CDCl}_{3}, 25^{\circ} \mathrm{C}\right): \delta=7.54-7.48(\mathrm{~m}, 3 \mathrm{H}$, $\left.\mathrm{H}_{m^{\prime}}, \mathrm{H}_{p^{\prime}}\right), 7.44\left(\mathrm{dt},{ }^{3} J_{\mathrm{o}, \mathrm{m}}=8.6 \mathrm{~Hz},{ }^{4} J_{\mathrm{o}, \mathrm{o}}={ }^{5} J_{\mathrm{o}, \mathrm{m}}=2.2 \mathrm{~Hz}, 2 \mathrm{H}, \mathrm{H}_{o}\right), 7.29\left(\mathrm{~m}, 2 \mathrm{H}, \mathrm{H}_{o^{\prime}}\right), 7.19(\mathrm{dt}$, ${ }^{3} J_{\mathrm{o}, \mathrm{m}}=8.6 \mathrm{~Hz},{ }^{4} J_{\mathrm{m}, \mathrm{m}}={ }^{5} J_{\mathrm{o}, \mathrm{m}}=2.2 \mathrm{~Hz}, 2 \mathrm{H}, \mathrm{H}_{m}$ ), $4.17\left(\mathrm{t},{ }^{3} J_{1^{\prime}, 2^{\prime}}=7.3 \mathrm{~Hz}, 2 \mathrm{H}, \mathrm{H}-1^{\prime}\right.$ ), 1.76 (quin, $\left.{ }^{3} J_{1^{\prime}, 2^{\prime}}={ }^{3} J_{2^{\prime}, 3^{\prime}}=7.3 \mathrm{~Hz}, 2 \mathrm{H}, \mathrm{H}-2^{\prime}\right), 1.24-1.12$ (m, $\left.4 \mathrm{H}, \mathrm{H}-3^{\prime}, \mathrm{H}-4{ }^{\prime}\right), 0.80$ (t, ${ }^{3} J_{4^{\prime}, 5^{\prime}}=6.5 \mathrm{~Hz}, 3 \mathrm{H}, \mathrm{H}-$ $\left.5^{\prime}\right) \mathrm{ppm} ;{ }^{13} \mathrm{C} \mathrm{NMR}\left(75 \mathrm{MHz}, \mathrm{CDCl}_{3}, 25{ }^{\circ} \mathrm{C}\right): \delta=143.1(\mathrm{C}-4), 133.7(\mathrm{C}-5), 133.3\left(\mathrm{C}_{p}\right), 129.8\left(\mathrm{C}_{p}\right.$, $\left.\mathrm{C}_{o}\right), 129.5\left(\mathrm{C}_{i p s o}\right), 129.4\left(\mathrm{C}_{m}{ }^{\prime}\right), 128.6\left(\mathrm{C}_{m}\right), 127.9\left(\mathrm{C}_{o}, \mathrm{C}_{i p s o^{\prime}}\right), 48.2\left(\mathrm{C}-1^{\prime}\right), 29.7\left(\mathrm{C}-2^{\prime}\right), 28.4\left(\mathrm{C}-3^{\prime}\right)$,

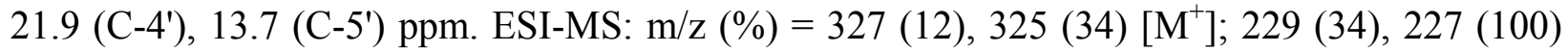

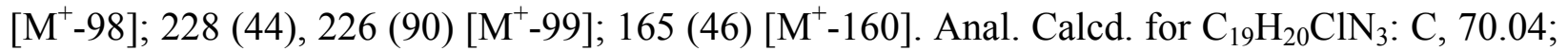
H, 6.19; N, 12.90. Found: C, 69.94; H, 6.18; N 12.65.

5-(4-Chlorophenyl)-1-pentyl-4-phenyl-1H-[1,2,3]triazole (10c). White solid (14.7 mg, 9\%); m.p. $110-112{ }^{\circ} \mathrm{C}$ (cyclohexane/AcOEt). $\left.{ }^{1} \mathrm{H} \mathrm{NMR} \mathrm{(300} \mathrm{MHz,} \mathrm{CDCl}_{3}, 25^{\circ} \mathrm{C}\right): \delta=7.52-7.47$ (m, 4 $\mathrm{H}, \mathrm{H}_{o}, \mathrm{H}_{m}$ ) , 7.29-7.24 (m, $\left.5 \mathrm{H}, \mathrm{H}_{o^{\prime}}, \mathrm{H}_{p}, \mathrm{H}_{m}\right), 4.18\left(\mathrm{t},{ }^{3} J_{1^{\prime}, 2^{\prime}}=7.4 \mathrm{~Hz}, 2 \mathrm{H}, \mathrm{H}-1^{\prime}\right), 1.78$ (2H, quin, $\left.{ }^{3} J_{1^{\prime}, 2^{\prime}}={ }^{3} J_{2^{\prime}, 3^{\prime}}=7.4 \mathrm{~Hz}, \mathrm{H}-2^{\prime}\right), 1.33-1.20\left(4 \mathrm{H}, \mathrm{m}, \mathrm{H}-3^{\prime}, \mathrm{H}-4^{\prime}\right), 0.83\left(3 \mathrm{H}, \mathrm{t},{ }^{3} J_{4^{\prime}, 5^{\prime}}=6.8 \mathrm{~Hz}, \mathrm{H}-5^{\prime}\right)$ ppm; ${ }^{13} \mathrm{C}$ NMR $\left(75 \mathrm{MHz}, \mathrm{CDCl}_{3}, 25{ }^{\circ} \mathrm{C}\right): \delta=144.4(\mathrm{C}-4), 135.9\left(\mathrm{C}_{p}\right), 132.4(\mathrm{C}-5), 131.3\left(\mathrm{C}_{o}{ }^{\prime}\right)$, 
$130.7\left(\mathrm{C}_{i p s o}\right), 129.7\left(\mathrm{C}_{m}{ }^{\prime}\right), 128.5\left(\mathrm{C}_{m}\right), 127.8\left(\mathrm{C}_{p}\right), 126.8\left(\mathrm{C}_{o}\right), 126.7\left(\mathrm{C}_{\text {ipso }}\right), 48.3\left(\mathrm{C}-1^{\prime}\right), 29.7(\mathrm{C}-$

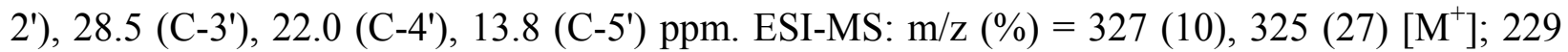

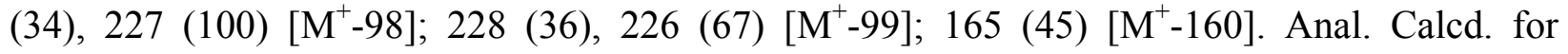
$\mathrm{C}_{19} \mathrm{H}_{20} \mathrm{ClN}_{3}$ : C, 70.04; H, 6.19; N, 12.90. Found: C, 70.29; H, 6.05; N 12.35.

4-(2,4-Dichlorophenyl)-2-pentyl-5-(4-methylphenyl)-2H-[1,2,3]triazole (11a). Colourless oil (136.6 mg, 73\%). ${ }^{1} \mathrm{H}$ NMR (300 MHz, $\left.\mathrm{CDCl}_{3}, 25{ }^{\circ} \mathrm{C}\right): \delta=7.43\left(\mathrm{~d},{ }^{4} J_{3^{\prime \prime}, 5^{\prime \prime}}=2.4 \mathrm{~Hz}, 1 \mathrm{H}, \mathrm{H}-3 "\right)$, $7.39\left(\mathrm{~d},{ }^{3} J_{5^{\prime \prime}, 6 "}=8.4 \mathrm{~Hz}, 1 \mathrm{H}, \mathrm{H}-6 "\right), 7.33-7.32$ (m, $\left.3 \mathrm{H}, \mathrm{H}-5 ", \mathrm{H}_{o}\right), 7.11\left(\mathrm{~d},{ }^{3} J_{\mathrm{o}, \mathrm{m}}=8.0 \mathrm{~Hz}, 2 \mathrm{H}\right.$, $\left.\mathrm{H}_{m}\right), 4.47\left(\mathrm{t},{ }^{3} J_{1^{\prime}, 2^{\prime}}=7.0 \mathrm{~Hz}, 2 \mathrm{H}, \mathrm{H}-1^{\prime}\right), 2.32\left(\mathrm{~s}, 3 \mathrm{H}, \mathrm{CH}_{3}\right), 2.05$ (quin, ${ }^{3} J_{1^{\prime}, 2^{\prime}}={ }^{3} J_{2^{\prime}, 3^{\prime}}=7.0 \mathrm{~Hz}, 2 \mathrm{H}$, H-2'), 1.38-1.35 (m, 4 H, H-3', H-4'), 0.91 (t, $\left.{ }^{3} J_{4^{\prime}, 5^{\prime}}=7.0 \mathrm{~Hz}, 3 \mathrm{H}, \mathrm{H}-5^{\prime}\right)$ ppm; ${ }^{13} \mathrm{C}$ NMR $(75$ $\left.\mathrm{MHz}, \mathrm{CDCl}_{3}, 25^{\circ} \mathrm{C}\right): \delta=145.2(\mathrm{C}-5), 140.4(\mathrm{C}-4), 138.1\left(\mathrm{C}_{p}\right), 132.8$ (C-1"), 132.7 (C-2"), 132.6 (C-4"), 131.8 (C-3"), 131.0 (C6"), 130.1 (C5"), $129.3\left(\mathrm{C}_{m}\right), 127.8\left(\mathrm{C}_{i p s o}\right), 126.6\left(\mathrm{C}_{o}\right), 55.3$ (C-1'), 29.5 (C-2'), 28.7 (C-3'), 22.1 (C-4'), $21.3\left(\mathrm{CH}_{3}\right), 13.9$ (C-5') ppm. ESI-MS: m/z (\%) = 377 (16), 375 (78), 373 (100) $\left[\mathrm{M}^{+}\right] ; 330$ (59) $\left[\mathrm{M}^{+}-43\right] ; 275$ (62) $\left[\mathrm{M}^{+}-98\right] ; 213$ (43) $\left[\mathrm{M}^{+}-161\right] ; 118$ (68) [ $\left.\mathrm{M}^{+}-255\right]$. Anal. Calcd. for $\mathrm{C}_{20} \mathrm{H}_{21} \mathrm{Cl}_{2} \mathrm{~N}_{3}$ : C, 64.18; H, 5.65; N, 11.23. Found: C, 64.21; H, 5.46; $\mathrm{N} 11.08$.

4-(4-Chlorophenyl)-2-heptyl-5-phenyl-2H-[1,2,3]triazole (12a). Colourless oil (113.2 mg, 64\%). ${ }^{1} \mathrm{H}$ NMR (200 MHz, $\left.\mathrm{CDCl}_{3}, 25^{\circ} \mathrm{C}\right): \delta=7.52-7.46\left(\mathrm{~m}, 4 \mathrm{H}, \mathrm{H}_{o}, \mathrm{H}_{o}\right)$ ) 7.37-7.30 (m, $5 \mathrm{H}$, $\mathrm{H}_{m}, \mathrm{H}_{p^{\prime}}, \mathrm{H}_{m}$ ), 4.44 (t, ${ }^{3} J_{1^{\prime}, 2^{\prime}}=7.2 \mathrm{~Hz}, 2 \mathrm{H}, \mathrm{H}-1^{\prime}$ ), 2.02 (quin, ${ }^{3} J_{1^{\prime}, 2^{\prime}}={ }^{3} J_{2^{\prime}, 3^{\prime}}=7.2 \mathrm{~Hz}, 2 \mathrm{H}, \mathrm{H}-2^{\prime}$ ), 1.39-1.26 (m, 8 H, H-3', H-4', H-5', H-6'), 0.86 (t, 3 H, $\left.{ }^{3} J_{6^{\prime}, 7^{\prime}}=7.2 \mathrm{~Hz}, \mathrm{H}-7^{\prime}\right)$ ppm; ${ }^{13} \mathrm{C}$ NMR (75 $\left.\mathrm{MHz}, \mathrm{CDCl}_{3}, 25^{\circ} \mathrm{C}\right): \delta=144.2(\mathrm{C}-5), 142.9(\mathrm{C}-4), 134.1\left(\mathrm{C}_{p}\right), 130.9\left(\mathrm{C}_{\text {ipso }}\right), 129.7\left(\mathrm{C}_{i p s o}\right), 129.4$ $\left(\mathrm{C}_{o}\right), 128.8\left(\mathrm{C}_{m}\right), 128.6\left(\mathrm{C}_{m^{\prime}}\right), 128.4\left(\mathrm{C}_{p^{\prime}}\right), 128.2\left(\mathrm{C}_{o^{\prime}}\right), 55.2\left(\mathrm{C}-1^{\prime}\right), 31.6\left(\mathrm{C}-5^{\prime}\right), 29.8\left(\mathrm{C}-2^{\prime}\right), 28.7$

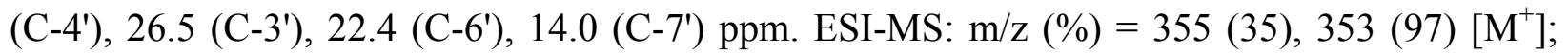

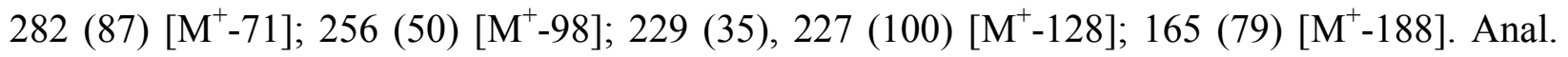
Calcd. for $\mathrm{C}_{21} \mathrm{H}_{24} \mathrm{ClN}_{3}$ : C, 71.27; H, 6.84; N, 11.87. Found: C, 71.63; H, 6.97; N 11.68.

4-(4-Chlorophenyl)-1-heptyl-5-phenyl-1H-[1,2,3]triazole (12b). White solid (31.8 mg, 18\%); m.p. 64-66 ${ }^{\circ} \mathrm{C}$ (cyclohexane/AcOEt). ${ }^{1} \mathrm{H}$ NMR (300 MHz, $\left.\mathrm{CDCl}_{3}, 25{ }^{\circ} \mathrm{C}\right): \delta=7.47-7.44(\mathrm{~m}, 3 \mathrm{H}$, $\left.\mathrm{H}_{m^{\prime}}, \mathrm{H}_{p^{\prime}}\right), 7.40\left(\mathrm{dt},{ }^{3} J_{\mathrm{o}, \mathrm{m}}=8.4 \mathrm{~Hz},{ }^{4} J_{\mathrm{o}, \mathrm{o}}={ }^{5} J_{\mathrm{o}, \mathrm{m}}=2.2 \mathrm{~Hz}, 2 \mathrm{H}, \mathrm{H}_{o}\right), 7.25-7.22\left(\mathrm{~m}, 2 \mathrm{H}, \mathrm{H}_{o^{\prime}}\right), 7.16$ $\left(\mathrm{dt},{ }^{3} J_{\mathrm{o}, \mathrm{m}}=8.4 \mathrm{~Hz},{ }^{4} J_{\mathrm{m}, \mathrm{m}}={ }^{5} J_{\mathrm{o}, \mathrm{m}}=2.2 \mathrm{~Hz}, 2 \mathrm{H}, \mathrm{H}_{m}\right), 4.12\left(\mathrm{t},{ }^{3} J_{1^{\prime}, 2^{\prime}}=7.2 \mathrm{~Hz}, 2 \mathrm{H}, \mathrm{H}-1^{\prime}\right), 1.17$ (quin, $\left.{ }^{3} J_{1^{\prime}, 2^{\prime}}={ }^{3} J_{2^{\prime}, 3^{\prime}}=7.2 \mathrm{~Hz}, 2 \mathrm{H}, \mathrm{H}-2^{\prime}\right), 1.18-1.13$ (m, $8 \mathrm{H}, \mathrm{H}-3$ ', H-4', H-5', H-6'), 0.78 (t, $3 \mathrm{H},{ }^{3} J_{6^{\prime}, 7^{\prime}}=$ $\left.7.2 \mathrm{~Hz}, \mathrm{H}_{-} 7^{\prime}\right) \mathrm{ppm} ;{ }^{13} \mathrm{C} \mathrm{NMR}\left(75 \mathrm{MHz}, \mathrm{CDCl}_{3}, 25{ }^{\circ} \mathrm{C}\right): \delta=143.1(\mathrm{C}-4), 133.8(\mathrm{C}-5), 133.4\left(\mathrm{C}_{p}\right)$, $129.9\left(\mathrm{C}_{o^{\prime}}\right), 129.8\left(\mathrm{C}_{p^{\prime}}\right), 129.6\left(\mathrm{C}_{i p s o}\right), 129.4\left(\mathrm{C}_{m^{\prime}}\right), 128.6\left(\mathrm{C}_{m}\right), 127.9\left(\mathrm{C}_{o}, \mathrm{C}_{i p s o}\right), 48.3\left(\mathrm{C}-1^{\prime}\right), 31.4$ (C-5'), 30.0 (C-2'), 28.5 (C-4'), 26.3 (C-3'), 22.4 (C-6'), 14.0 (C-7') ppm. ESI-MS: m/z (\%) = 355 (10), 353 (28) $\left[\mathrm{M}^{+}\right] ; 229$ (35), 227 (100) [ $\left.\mathrm{M}^{+}-128\right] ; 228$ (45), 226 (86) [ $\mathrm{M}^{+}$-129]. Anal. Calcd. for $\mathrm{C}_{21} \mathrm{H}_{24} \mathrm{ClN}_{3}$ : C, 71.27; H, 6.84; N, 11.87. Found: C, 71.32; H, 7.91; N 11.80.

5-(4-Chlorophenyl)-1-heptyl-4-phenyl-1H-[1,2,3]triazole (12c). Colourless oil (15.9 mg, 9\%). ${ }^{1} \mathrm{H}$ NMR (300 MHz, $\left.\mathrm{CDCl}_{3}, 25^{\circ} \mathrm{C}\right): \delta=7.46-7.41\left(\mathrm{~m}, 4 \mathrm{H}, \mathrm{H}_{o}, \mathrm{H}_{m}\right.$ ) $)$ 7.21-7.17 (m, $5 \mathrm{H}, \mathrm{H}_{o}, \mathrm{H}_{p}$, $\mathrm{H}_{m}$ ), $4.12\left(\mathrm{t},{ }^{3} J_{1^{\prime}, 2^{\prime}}=7.2 \mathrm{~Hz}, 2 \mathrm{H}, \mathrm{H}-1^{\prime}\right.$ ), 1.71 (quin, ${ }^{3} J_{1^{\prime}, 2^{\prime}}={ }^{3} J_{2^{\prime}, 3^{\prime}}=7.2 \mathrm{~Hz}, 2 \mathrm{H}, \mathrm{H}-2^{\prime}$ ), 1.18-1.14 (m, 8 H, H-3', H-4', H-5', H-6'), 0.77 (t, $\left.3 \mathrm{H},{ }^{3} J_{6^{\prime}, 7^{\prime}}=7.2 \mathrm{~Hz}, \mathrm{H}-7^{\prime}\right)$ ppm; ${ }^{13} \mathrm{C}$ NMR (75 MHz, $\left.\mathrm{CDCl}_{3}, 25^{\circ} \mathrm{C}\right): \delta=144.3(\mathrm{C}-4), 135.9\left(\mathrm{C}_{p}\right), 132.5(\mathrm{C}-5), 131.3\left(\mathrm{C}_{o}\right), 130.7\left(\mathrm{C}_{i p s o}\right), 129.8\left(\mathrm{C}_{m^{\prime}}\right)$, $128.5\left(\mathrm{C}_{m}\right), 127.8\left(\mathrm{C}_{p}\right), 126.9\left(\mathrm{C}_{o}\right), 126.8\left(\mathrm{C}_{i p s o}\right), 48.3\left(\mathrm{C}-1^{\prime}\right), 31.4\left(\mathrm{C}-5^{\prime}\right), 30.0\left(\mathrm{C}-2^{\prime}\right), 28.5\left(\mathrm{C}-4^{\prime}\right)$, 
26.4 (C-3'), 22.4 (C-6'), 14.0 (C-7') ppm. ESI-MS: m/z (\%) = 355 (7), $353(19)\left[\mathrm{M}^{+}\right] ; 229(35)$, 227 (100) [ $\left.\mathrm{M}^{+}-128\right] ; 228$ (38), $226(65)$ [ $\left.\mathrm{M}^{+}-129\right]$. Anal. Calcd. for $\mathrm{C}_{21} \mathrm{H}_{24} \mathrm{ClN}_{3}$ : C, 71.27; $\mathrm{H}$, 6.84; N, 11.87. Found: C, 71.40; H, 6.80; N 11.98 .

2-(4-Bromobenzyl)-4-(p-chlorophenyl)-5-phenyl-2H-[1,2,3]triazole (13a). White solid (131.6 mg, 62\%); m.p. $104-106{ }^{\circ} \mathrm{C}$ (cyclohexane). ${ }^{1} \mathrm{H}$ NMR (300 MHz, $\left.\mathrm{CDCl}_{3}, 25{ }^{\circ} \mathrm{C}\right): \delta=7.52-7.45$ $\left(\mathrm{m}, 6 \mathrm{H}, \mathrm{H}_{o}, \mathrm{H}_{o^{\prime}}, \mathrm{H}_{m^{\prime \prime}}\right)$, 7.38-7.34 (m, $\left.3 \mathrm{H}, \mathrm{H}_{m^{\prime}}, \mathrm{H}_{p^{\prime}}\right), 7.33-7.29\left(\mathrm{~m}, 4 \mathrm{H}, \mathrm{H}_{m}, \mathrm{H}_{o^{\prime \prime}}\right), 5.57(\mathrm{~s}, 2 \mathrm{H}$, $\left.\mathrm{CH}_{2}\right)$ ppm; ${ }^{13} \mathrm{C}$ NMR $\left(75 \mathrm{MHz}, \mathrm{CDCl}_{3}, 25^{\circ} \mathrm{C}\right): \delta=145.1(\mathrm{C}-5), 143.9(\mathrm{C}-4), 134.3\left(\mathrm{C}_{\text {ipso }}\right), 134.0$ $\left(\mathrm{C}_{p}\right), 131.9\left(\mathrm{C}_{m^{\prime \prime}}\right), 130.5\left(\mathrm{C}_{i p s o^{\prime}}\right), 129.9\left(\mathrm{C}_{o^{\prime \prime}}\right), 129.5\left(\mathrm{C}_{o}\right), 129.3\left(\mathrm{C}_{i p s o}\right), 128.8\left(\mathrm{C}_{m}\right), 128.7\left(\mathrm{C}_{m^{\prime}}\right)$, $128.6\left(\mathrm{C}_{p^{\prime}}\right), 128.2\left(\mathrm{C}_{o^{\prime}}\right), 122.5\left(\mathrm{C}_{p^{\prime \prime}}\right), 58.1\left(\mathrm{CH}_{2}\right) \mathrm{ppm} . \mathrm{ESI}-\mathrm{MS}: \mathrm{m} / \mathrm{z}(\%)=427(36), 425(97), 423$ (78) $\left[\mathrm{M}^{+}\right] ; 228$ (47), $226(100)\left[\mathrm{M}^{+}-199\right] ; 171(82), 169(80)\left[\mathrm{M}^{+}-255\right]$. Anal. Calcd. for $\mathrm{C}_{21} \mathrm{H}_{15} \mathrm{ClBrN}_{3}$ : C, 59.39; H, 3.56; N, 9.89. Found: C, 59.10; H, 3.72; N 9.38.

1-(4-Bromobenzyl)-4-(p-chlorophenyl)-5-phenyl-1H-[1,2,3]triazole (13b). White solid (29.7 mg, 14\%); m.p. $120-122{ }^{\circ} \mathrm{C}$ (cyclohexane). ${ }^{1} \mathrm{H}$ NMR (300 MHz, $\left.\mathrm{CDCl}_{3}, 25^{\circ} \mathrm{C}\right): \delta=7.49-7.44$ $\left(\mathrm{m}, 5 \mathrm{H}, \mathrm{H}_{m^{\prime}}, \mathrm{H}_{m^{\prime \prime}}, \mathrm{H}_{p^{\prime}}\right), 7.38\left(\mathrm{dt},{ }^{3} J_{\mathrm{o}, \mathrm{m}}=8.4 \mathrm{~Hz},{ }^{4} J_{\mathrm{o}, \mathrm{o}}={ }^{5} J_{\mathrm{o}, \mathrm{m}}=2.4 \mathrm{~Hz}, 2 \mathrm{H}, \mathrm{H}_{o}\right), 7.22\left(\mathrm{dt},{ }^{3} J_{\mathrm{o}, \mathrm{m}}=\right.$ $\left.8.4 \mathrm{~Hz},{ }^{4} J_{\mathrm{m}, \mathrm{m}}={ }^{5} J_{\mathrm{o}, \mathrm{m}}=2.4 \mathrm{~Hz}, 2 \mathrm{H}, \mathrm{H}_{m}\right), 7.15-7.11\left(\mathrm{~m}, 2 \mathrm{H}, \mathrm{H}_{o^{\prime}}\right), 6.89\left(\mathrm{~d},{ }^{3} J_{\mathrm{o}^{\prime \prime}, \mathrm{m}^{\prime \prime}}=8.4 \mathrm{~Hz}, 2 \mathrm{H}\right.$, $\left.\mathrm{H}_{o^{\prime \prime}}\right), 5.34$ (s, $\left.2 \mathrm{H}, \mathrm{CH}_{2}\right) \mathrm{ppm} ;{ }^{13} \mathrm{C} \mathrm{NMR}\left(75 \mathrm{MHz}, \mathrm{CDCl}_{3}, 25^{\circ} \mathrm{C}\right): \delta=144.1(\mathrm{C}-4), 134.1\left(\mathrm{C}_{\text {ipso" }}\right)$, $133.9(\mathrm{C}-5), 133.6\left(\mathrm{C}_{p}\right), 131.9\left(\mathrm{C}_{m^{\prime \prime}}\right), 130.0\left(\mathrm{C}_{p^{\prime}}\right), 129.9\left(\mathrm{C}_{o^{\prime}}, \mathrm{C}_{i p s o}\right), 129.4\left(\mathrm{C}_{m^{\prime}}\right), 129.2\left(\mathrm{C}_{o^{\prime \prime}}\right), 128.7$ $\left(\mathrm{C}_{m}\right), 127.9\left(\mathrm{C}_{o}\right), 127.4\left(\mathrm{C}_{i p s o^{\prime}}\right), 122.4\left(\mathrm{C}_{p^{\prime \prime}}\right), 51.8\left(\mathrm{CH}_{2}\right)$ ppm. ESI-MS: m/z $(\%)=425(6)\left[\mathrm{M}^{+}\right]$; 228 (37), 226 (100) [ $\left.\mathrm{M}^{+}-199\right] ; 171$ (52), 169 (54) [ $\left.\mathrm{M}^{+}-255\right]$. Anal. Calcd. for $\mathrm{C}_{21} \mathrm{H}_{15} \mathrm{ClBrN}_{3}: \mathrm{C}$, 59.39; H, 3.56; N, 9.89. Found: C, 59.42; H, 3.91; N 9.53.

1-(4-Bromobenzyl)-5-(p-chlorophenyl)-4-phenyl-1H-[1,2,3]triazole (13c). White solid (40.3 mg, 19\%); m.p. $164-166{ }^{\circ} \mathrm{C}$ (cyclohexane). ${ }^{1} \mathrm{H}$ NMR (300 MHz, $\left.\mathrm{CDCl}_{3}, 25{ }^{\circ} \mathrm{C}\right): \delta=7.54-7.50$ $\left(\mathrm{m}, 2 \mathrm{H}, \mathrm{H}_{m^{\prime \prime}}\right)$, 7.43-7.39 (m, $\left.4 \mathrm{H}, \mathrm{H}_{o}, \mathrm{H}_{m^{\prime}}\right)$ 7.31-7.26 (m, $\left.3 \mathrm{H}, \mathrm{H}_{m}, \mathrm{H}_{p}\right), 7.06\left(\mathrm{dt},{ }^{3} J_{\mathrm{o}^{\prime}, \mathrm{m}^{\prime}}=8.6 \mathrm{~Hz}\right.$, $\left.{ }^{4} J_{\mathrm{o}^{\prime}, \mathrm{o}^{\prime}}={ }^{5} J_{\mathrm{o}^{\prime}, \mathrm{m}^{\prime}}=2.0 \mathrm{~Hz}, 2 \mathrm{H}, \mathrm{H}_{o^{\prime}}\right) 6.92\left(\mathrm{~d},{ }^{3} J_{\mathrm{o}^{\prime \prime}, \mathrm{m}^{\prime \prime}}=8.5 \mathrm{~Hz}, 2 \mathrm{H}, \mathrm{H}_{o^{\prime}}\right), 5.35\left(\mathrm{~s}, 2 \mathrm{H}, \mathrm{CH}_{2}\right) \mathrm{ppm} ;{ }^{13} \mathrm{C}$ NMR (75 MHz, $\left.\mathrm{CDCl}_{3}, 25^{\circ} \mathrm{C}\right): \delta=145.0(\mathrm{C}-4), 136.3\left(\mathrm{C}_{p}\right), 134.1(\mathrm{C}-5), 134.0\left(\mathrm{C}_{\text {ipso" }}\right), 131.9$ $\left(\mathrm{C}_{m^{\prime \prime}}\right), 131.4\left(\mathrm{C}_{o^{\prime}}\right), 130.4\left(\mathrm{C}_{i p s o}\right), 129.7\left(\mathrm{C}_{m}\right), 129.1\left(\mathrm{C}_{o^{\prime \prime}}\right), 128.6\left(\mathrm{C}_{m}\right), 128.0\left(\mathrm{C}_{p}\right), 126.7\left(\mathrm{C}_{o}\right)$, $126.1\left(\mathrm{C}_{\text {ipso }}\right), 122.3\left(\mathrm{C}_{p^{\prime \prime}}\right), 58.1\left(\mathrm{CH}_{2}\right)$ ppm. ESI-MS: m/z $(\%)=425(10)\left[\mathrm{M}^{+}\right] ; 228(39), 226$ (100) [ $\left.\mathrm{M}^{+}-199\right] ; 171$ (49), 169 (51) $\left[\mathrm{M}^{+}-255\right]$. Anal. Calcd. for $\mathrm{C}_{21} \mathrm{H}_{15} \mathrm{ClBrN}_{3}$ : C, 59.39; H, 3.56; N, 9.89. Found: C, 59.22; H, 3.88; N 9.77.

2-Benzyl-4-(4-chlorophenyl)-5-phenyl-2H-[1,2,3]triazole (14a). White solid (110.7 mg, 64\%); m.p. $110-112{ }^{\circ} \mathrm{C}$ (cyclohexane). ${ }^{1} \mathrm{H}$ NMR $\left(500 \mathrm{MHz}, \mathrm{CDCl}_{3}, 25^{\circ} \mathrm{C}\right): \delta=7.52-7.45$ (m, $4 \mathrm{H}, \mathrm{H}_{o}$, $\left.\mathrm{H}_{o^{\prime}}\right)$, 7.46-7.42 (m, $\left.2 \mathrm{H}, \mathrm{H}_{o^{\prime \prime}}\right), 7.40-7.32\left(\mathrm{~m}, 8 \mathrm{H}, \mathrm{H}_{m^{\prime \prime}}, \mathrm{H}_{p^{\prime \prime}}, \mathrm{H}_{m^{\prime}}, \mathrm{H}_{p^{\prime}}, \mathrm{H}_{m}\right), 5.62\left(\mathrm{~s}, 2 \mathrm{H}, \mathrm{CH}_{2}\right)$ ppm; ${ }^{13} \mathrm{C}$ NMR $\left(75 \mathrm{MHz}, \mathrm{CDCl}_{3}, 25{ }^{\circ} \mathrm{C}\right): \delta=145.1(\mathrm{C}-5), 143.9(\mathrm{C}-4), 135.4\left(\mathrm{C}_{i p s o}\right), 134.5\left(\mathrm{C}_{p}\right)$, $131.0\left(\mathrm{C}_{i p s o^{\prime}}\right), 129.8\left(\mathrm{C}_{i p s o}, \mathrm{C}_{o}\right), 129.1\left(\mathrm{C}_{o^{\prime \prime}}\right), 129.0\left(\mathrm{C}_{m}\right), 128.9\left(\mathrm{C}_{m^{\prime}}\right), 128.7\left(\mathrm{C}_{p^{\prime \prime}}\right), 128.6\left(\mathrm{C}_{p^{\prime}}\right)$, $128.5\left(\mathrm{C}_{o^{\prime}}\right) 128.4\left(\mathrm{C}_{m^{\prime \prime}}\right), 59.0\left(\mathrm{CH}_{2}\right)$ ppm. ESI-MS: m/z (\%) = $347(58), 345(100)\left[\mathrm{M}^{+}\right] ; 226(79)$ [ $\left.\mathrm{M}^{+}-119\right]$; 91 (99) [ $\left.\mathrm{M}^{+}-254\right]$. Anal. Calcd. for $\mathrm{C}_{21} \mathrm{H}_{16} \mathrm{ClN}_{3}$ : $\mathrm{C}, 72.93 ; \mathrm{H}, 4.66 ; \mathrm{N}, 12.15$. Found: C, $72.68 ; \mathrm{H}, 4.75 ; \mathrm{N} 12.10$.

1-Benzyl-4-(4-chlorophenyl)-5-phenyl-1H-[1,2,3]triazole (14b). White solid (41.4 mg, 24\%); m.p. $145-147{ }^{\circ} \mathrm{C}$ (cyclohexane). ${ }^{1} \mathrm{H}$ NMR (500 $\left.\mathrm{MHz}, \mathrm{CDCl}_{3}, 25^{\circ} \mathrm{C}\right): \delta=7.51-7.46\left(\mathrm{~m}, 3 \mathrm{H}, \mathrm{H}_{m^{\prime}}\right.$, $\left.\mathrm{H}_{p^{\prime}}\right)$, 7.44-7.40 (m, $\left.2 \mathrm{H}, \mathrm{H}_{o}\right), 7.26-7.20\left(\mathrm{~m}, 5 \mathrm{H}, \mathrm{H}_{m^{\prime \prime}}, \mathrm{H}_{p^{\prime \prime}}, \mathrm{H}_{m^{\prime}}\right)$, 7.14-7.10 (m, $\left.2 \mathrm{H}, \mathrm{H}_{o^{\prime}}\right), 7.03-7.00$ 
$\left(\mathrm{m}, 2 \mathrm{H}, \mathrm{H}_{o^{\prime \prime}}\right), 5.40\left(\mathrm{~s}, 2 \mathrm{H}, \mathrm{CH}_{2}\right)$ ppm; $\left.{ }^{13} \mathrm{C} \mathrm{NMR} \mathrm{(125} \mathrm{MHz,} \mathrm{CDCl}_{3}, 25{ }^{\circ} \mathrm{C}\right): \delta=143.5(\mathrm{C}-4)$, $135.2\left(\mathrm{C}_{\text {ipso" }}\right), 134.0(\mathrm{C}-5), 133.1\left(\mathrm{C}_{p}\right), 130.0\left(\mathrm{C}_{i p s o}, \mathrm{C}_{o}\right), 129.8\left(\mathrm{C}_{p^{\prime}}\right), 129.4\left(\mathrm{C}_{o^{\prime \prime}}\right), 129.3\left(\mathrm{C}_{m^{\prime}}\right)$, 128.6 $\left(\mathrm{C}_{m}, \mathrm{C}_{m^{\prime \prime}}\right), 128.2\left(\mathrm{C}_{p^{\prime \prime}}\right), 127.9\left(\mathrm{C}_{i p s o}\right), 127.5\left(\mathrm{C}_{o^{\prime \prime}}\right), 52.1\left(\mathrm{CH}_{2}\right)$ ppm. ESI-MS: m/z $(\%)=347$

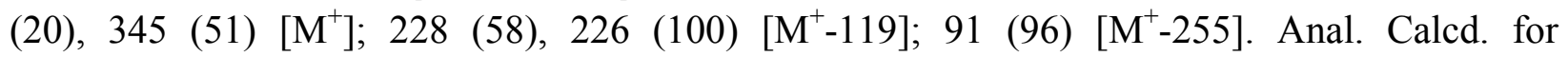
$\mathrm{C}_{21} \mathrm{H}_{16} \mathrm{ClN}_{3}$ : C, 72.93; H, 4.66; N, 12.15. Found: C, 72.82; H, 4.90; N 11.93 .

1-Benzyl-5-(4-chlorophenyl)-4-phenyl-1H-[1,2,3]triazole (14c). White solid (15.5 mg, 9\%); m.p. $177-179{ }^{\circ} \mathrm{C}$ (cyclohexane). ${ }^{1} \mathrm{H}$ NMR $\left(200 \mathrm{MHz}, \mathrm{CDCl}_{3}, 25^{\circ} \mathrm{C}\right): \delta=7.57-7.52\left(\mathrm{~m}, 2 \mathrm{H}, \mathrm{H}_{o}\right)$, 7.43-7.39 (d, $\left.{ }^{3} J_{\mathrm{m}^{\prime}, \mathrm{o}^{\prime}}=8.4 \mathrm{~Hz}, 2 \mathrm{H}, \mathrm{H}_{m^{\prime}}\right), 7.30-7.28\left(\mathrm{~m}, 6 \mathrm{H}, \mathrm{H}_{o^{\prime \prime}}, \mathrm{H}_{p^{\prime \prime}}, \mathrm{H}_{m}, \mathrm{H}_{p}\right)$, 7.10-7.06 (m, $4 \mathrm{H}$, $\left.\mathrm{H}_{o^{\prime}}, \mathrm{H}_{m^{\prime \prime}}\right), 5.43\left(\mathrm{~s}, 2 \mathrm{H}, \mathrm{CH}_{2}\right)$ ppm; ${ }^{13} \mathrm{C} \mathrm{NMR}\left(125 \mathrm{MHz}, \mathrm{CDCl}_{3}, 25{ }^{\circ} \mathrm{C}\right): \delta=144.8(\mathrm{C}-4), 136.0$ $\left(\mathrm{C}_{p^{\prime}}\right), 135.2\left(\mathrm{C}_{i p s o^{\prime \prime}}\right), 132.7(\mathrm{C}-5), 131.4\left(\mathrm{C}_{o}\right), 130.6\left(\mathrm{C}_{i p s o}\right), 129.5\left(\mathrm{C}_{m^{\prime}}\right), 128.8\left(\mathrm{C}_{m^{\prime \prime}}\right), 128.5\left(\mathrm{C}_{m}\right)$, $127.9\left(\mathrm{C}_{p}\right), 127.3\left(\mathrm{C}_{p^{\prime \prime}}\right), 127.3\left(\mathrm{C}_{o^{\prime \prime}}\right), 126.7\left(\mathrm{C}_{o}\right), 126.3\left(\mathrm{C}_{i p s o^{\prime}}\right), 52.1\left(\mathrm{CH}_{2}\right) \mathrm{ppm} . \mathrm{ESI}-\mathrm{MS}: \mathrm{m} / \mathrm{z}$ $(\%)=347(14), 345(36)\left[\mathrm{M}^{+}\right] ; 228(43), 226(95)\left[\mathrm{M}^{+}-119\right] ; 91$ (100) [ $\left.\mathrm{M}^{+}-255\right]$. Anal. Calcd. for $\mathrm{C}_{21} \mathrm{H}_{16} \mathrm{ClN}_{3}$ : C, 72.93; H, 4.66; N, 12.15. Found: C, 72.98; H, 4.94; N 11.83 .

2-Benzyl-4-(4-bromo-2-fluorophenyl)-2H-[1,2,3]triazole (15a). White solid (73.1 mg, 44\%); m.p. $104-106{ }^{\circ} \mathrm{C}$ (cyclohexane). ${ }^{1} \mathrm{H}$ NMR $\left(500 \mathrm{MHz}, \mathrm{CDCl}_{3}, 25{ }^{\circ} \mathrm{C}\right): \delta=7.97\left(\mathrm{~d},{ }^{5} J_{5, \mathrm{~F}}=3.9 \mathrm{~Hz}\right.$, $1 \mathrm{H}, \mathrm{H}-5), 7.88$ (t, $\left.{ }^{3} J_{5^{\prime}, 6^{\prime}}={ }^{4} J_{6^{\prime}, \mathrm{F}}=8.3 \mathrm{~Hz}, 1 \mathrm{H}, \mathrm{H}-6^{\prime}\right), 7.40-7.34$ (m, $\left.7 \mathrm{H}, \mathrm{H}_{o}, \mathrm{H}_{m}, \mathrm{H}_{p}, \mathrm{H}-3^{\prime}, \mathrm{H}-5^{\prime}\right)$, $5.60\left(\mathrm{~s}, 2 \mathrm{H}, \mathrm{CH}_{2}\right) \mathrm{ppm} ;{ }^{13} \mathrm{C} \mathrm{NMR}\left(50 \mathrm{MHz}, \mathrm{CDCl}_{3}, 25{ }^{\circ} \mathrm{C}\right): \delta=159.8\left(\mathrm{~d},{ }^{1} J_{2^{\prime}, \mathrm{F}}=254.1 \mathrm{~Hz}, \mathrm{C}-2^{\prime}\right)$, $141.9\left(\mathrm{~d},{ }^{3} J_{4, \mathrm{~F}}=1.5 \mathrm{~Hz}, \mathrm{C}-4\right), 135.3\left(\mathrm{C}_{i p s o}\right), 134.4\left(\mathrm{~d},{ }^{4} J_{5, \mathrm{~F}}=11.4 \mathrm{~Hz}, \mathrm{C}-5\right), 129.5\left(\mathrm{~d},{ }^{3} J_{6, \mathrm{~F}}=4.4\right.$ $\left.\mathrm{Hz}, \mathrm{C}-6^{\prime}\right), 129.1\left(\mathrm{C}_{o}\right) 128.7\left(\mathrm{C}_{p}\right), 128.3\left(\mathrm{C}_{m}\right), 128.1$ (d, $\left.{ }^{4} J_{5^{\prime}, \mathrm{F}}=3.8 \mathrm{~Hz}, \mathrm{C}-5^{\prime}\right), 122.3\left(\mathrm{~d},{ }^{3} J_{4^{\prime}, \mathrm{F}}=9.9\right.$ $\left.\mathrm{Hz}, \mathrm{C}-4^{\prime}\right), 119.9$ (d, $\left.{ }^{2} J_{3^{\prime}, \mathrm{F}}=25.2 \mathrm{~Hz}, \mathrm{C}-3^{\prime}\right), 117.8\left(\mathrm{~d},{ }^{2} J_{1^{\prime}, \mathrm{F}}=13.0 \mathrm{~Hz}, \mathrm{C}-1^{\prime}\right), 59.1\left(\mathrm{CH}_{2}\right) \mathrm{ppm}$. ESI-

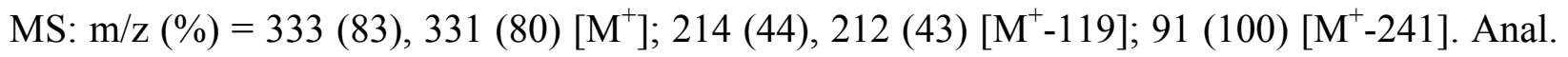
Calcd. for $\mathrm{C}_{15} \mathrm{H}_{11} \mathrm{BrFN}_{3}$ : C, 54.24; H, 3.34; N, 12.65. Found: C, 53.98; H, 3.21; N 12.42.

1-Benzyl-4-(4-bromo-2-fluorophenyl)-1H-[1,2,3]triazole (15b). White solid (89.7 mg, 54\%); m.p. $146-148{ }^{\circ} \mathrm{C}$ (cyclohexane). ${ }^{1} \mathrm{H}$ NMR (500 $\left.\mathrm{MHz}, \mathrm{CDCl}_{3}, 25{ }^{\circ} \mathrm{C}\right): \delta=8.17\left(\mathrm{dd},{ }^{3} J_{6^{\prime}, 5^{\prime}}=8.3\right.$ $\left.\mathrm{Hz},{ }^{4} J_{6^{\prime}, \mathrm{F}}=7.8 \mathrm{~Hz}, 1 \mathrm{H}, \mathrm{H}-6^{\prime}\right), 7.83\left(\mathrm{~d},{ }^{5} J_{5, \mathrm{~F}}=3.4 \mathrm{~Hz}, 1 \mathrm{H}, \mathrm{H}-5\right), 7.39-7.34$ (m, $4 \mathrm{H}, \mathrm{H}-5^{\prime}, \mathrm{H}_{m}$, $\left.\mathrm{H}_{p}\right)$, 7.30-7.24 (m, $3 \mathrm{H}, \mathrm{H}-3$ ', $\left.\mathrm{H}_{o}\right), 5.57$ (s, $\left.2 \mathrm{H}, \mathrm{CH}_{2}\right)$ ppm; ${ }^{13} \mathrm{C} \mathrm{NMR}\left(50 \mathrm{MHz}, \mathrm{CDCl}_{3}, 25{ }^{\circ} \mathrm{C}\right): \delta$ $=158.7\left(\mathrm{~d},{ }^{1} J_{2^{\prime}, \mathrm{F}}=251.7 \mathrm{~Hz}, \mathrm{C}-2^{\prime}\right), 140.6\left(\mathrm{~d},{ }^{3} J_{4, \mathrm{~F}}=3.1 \mathrm{~Hz}, \mathrm{C}-4\right), 134.5\left(\mathrm{C}_{i p s o}\right), 129.1\left(\mathrm{C}_{m}\right), 128.8$ $\left(\mathrm{C}_{p}\right), 128.7\left(\mathrm{~d},{ }^{3} J_{6^{\prime}, \mathrm{F}}=4.6 \mathrm{~Hz}, \mathrm{C}-6^{\prime}\right), 127.9\left(\mathrm{C}_{o}, \mathrm{C}-5^{\prime}\right), 122.6\left(\mathrm{~d},{ }^{4} J_{5, \mathrm{~F}}=12.2 \mathrm{~Hz}, \mathrm{C}-5\right), 121.6(\mathrm{~d}$, $\left.{ }^{3} J_{4^{\prime}, \mathrm{F}}=11.0 \mathrm{~Hz}, \mathrm{C}-4^{\prime}\right), 119.2\left(\mathrm{~d},{ }^{2} J_{3^{\prime}, \mathrm{F}}=25.2 \mathrm{~Hz}, \mathrm{C}-3^{\prime}\right), 117.8\left(\mathrm{~d},{ }^{2} J_{1^{\prime}, \mathrm{F}}=12.9 \mathrm{~Hz}, \mathrm{C}-1^{\prime}\right), 54.2$

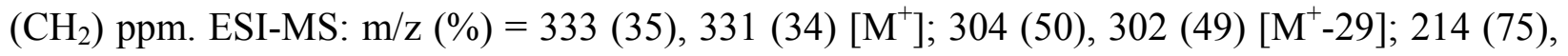
212 (73) $\left[\mathrm{M}^{+}-119\right] ; 197$ (50) $\left[\mathrm{M}^{+}-137\right]$; 91 (100) [ $\left.\mathrm{M}^{+}-241\right]$. Anal. Calcd. for $\mathrm{C}_{15} \mathrm{H}_{11} \mathrm{BrFN}_{3}$ : C, 54.24; H, 3.34; N, 12.65. Found: C, 54.15; H, 3.45; N 12.38 .

4-(4-Bromo-2-fluorophenyl)-2-(ethoxycarbonyl)methyl-2H[1,2,3]-triazole (16a). White solid (90.2, 55\%); m.p. $107-109{ }^{\circ} \mathrm{C}$ (cyclohexane/Et $\left.2 \mathrm{O}\right) .{ }^{1} \mathrm{H}$ NMR $\left(200 \mathrm{MHz}, \mathrm{CDCl}_{3}, 25{ }^{\circ} \mathrm{C}\right): \delta=8.04$ $\left(\mathrm{d},{ }^{5} J_{5, \mathrm{~F}}=4.0 \mathrm{~Hz}, 1 \mathrm{H}, \mathrm{H}-5\right), 7.89\left(\mathrm{dd},{ }^{3} J_{6^{\prime}, 5^{\prime}}=8.5 \mathrm{~Hz},{ }^{4} J_{6^{\prime}, \mathrm{F}}=7.2 \mathrm{~Hz}, 1 \mathrm{H}, \mathrm{H}-6^{\prime}\right), 7.37\left(\mathrm{~d},{ }^{3} J_{5^{\prime}, 6^{\prime}}=\right.$ $\left.8.5 \mathrm{~Hz},{ }^{3} J_{3^{\prime}, \mathrm{F}}=8.5 \mathrm{~Hz}, 2 \mathrm{H}, \mathrm{H}-5^{\prime}, \mathrm{H}-3^{\prime}\right), 5.27$ (s, $\left.2 \mathrm{H}, \mathrm{CH}_{2}\right), 4.28$ (q, $\left.{ }^{3} J_{1^{\prime \prime}, 2^{\prime \prime}}=7.0 \mathrm{~Hz}, 2 \mathrm{H}, \mathrm{H}-1^{\prime \prime}\right)$, $1.30\left(\mathrm{t},{ }^{3} J_{1 ", 2 "}=7.0 \mathrm{~Hz}, 3 \mathrm{H}, \mathrm{H}-2^{\prime \prime}\right)$ ppm. ${ }^{13} \mathrm{C} \mathrm{NMR}\left(50 \mathrm{MHz}, \mathrm{CDCl}_{3}, 25{ }^{\circ} \mathrm{C}\right): \delta=166.5(\mathrm{CO})$, $159.5\left(\mathrm{~d},{ }^{1} J_{2^{\prime}, \mathrm{F}}=254.7 \mathrm{~Hz}, \mathrm{C}-2^{\prime}\right), 142.3\left(\mathrm{~d},{ }^{3} J_{4, \mathrm{~F}}=2.3 \mathrm{~Hz}, \mathrm{C}-4\right), 134.7\left(\mathrm{~d},{ }^{4} J_{5, \mathrm{~F}}=12.4 \mathrm{~Hz}, \mathrm{C}-5\right)$, $129.3\left(\mathrm{~d},{ }^{3} J_{6^{\prime}, \mathrm{F}}=4.6 \mathrm{~Hz}, \mathrm{C}-6^{\prime}\right), 127.9\left(\mathrm{~d},{ }^{4} J_{5^{\prime}, \mathrm{F}}=4.1 \mathrm{~Hz}, \mathrm{C}-5^{\prime}\right), 122.3\left(\mathrm{~d},{ }^{3} J_{4^{\prime}, \mathrm{F}}=9.2 \mathrm{~Hz}, \mathrm{C}-4^{\prime}\right)$, $119.6\left(\mathrm{~d},{ }^{2} J_{3^{\prime}, \mathrm{F}}=25.1 \mathrm{~Hz}, \mathrm{C}-3^{\prime}\right), 117.2\left(\mathrm{~d},{ }^{2} J_{1^{\prime}, \mathrm{F}}=12.0 \mathrm{~Hz}, \mathrm{C}-1^{\prime}\right), 62.1\left(\mathrm{CH}_{2}\right), 55.6\left(\mathrm{C}-1^{\prime \prime}\right), 14.0(\mathrm{C}-$ 
2") ppm. ESI-MS: m/z (\%) = 329 (100), $327(99)\left[\mathrm{M}^{+}\right] ; 256$ (93), 258 (92) $\left[\mathrm{M}^{+}-73\right] ; 202$ (34), 200 (43) $\left[\mathrm{M}^{+}-129\right]$. Anal. Calcd. for $\mathrm{C}_{12} \mathrm{H}_{11} \mathrm{BrFN}_{3} \mathrm{O}_{2}$ : C, 43.92; H, 3.38; N, 12.81. Found: $\mathrm{C}$, $43.74 ; \mathrm{H}, 3.50 ; \mathrm{N} 12.68$.

4-(4-Bromo-2-fluorophenyl)-1-(ethoxycarbonyl)methyl-1H-[1,2,3]triazole (16b). White solid (29.5, 18\%); m.p. $115-117^{\circ} \mathrm{C}$ (cyclohexane/ $\left.\mathrm{Et}_{2} \mathrm{O}\right) .{ }^{1} \mathrm{H}$ NMR $\left(200 \mathrm{MHz}, \mathrm{CDCl}_{3}, 25^{\circ} \mathrm{C}\right): \delta=8.17$ $\left(\mathrm{t},{ }^{3} J_{5^{\prime}, 6^{\prime}}={ }^{4} J_{6^{\prime}, \mathrm{F}}=8.2 \mathrm{~Hz}, 1 \mathrm{H}, \mathrm{H}-6^{\prime}\right), 8.18\left(\mathrm{~d},{ }^{5} J_{5, \mathrm{~F}}=3.6 \mathrm{~Hz}, 1 \mathrm{H}, \mathrm{H}-5\right), 7.41-7.33\left(\mathrm{~m}, 2 \mathrm{H}, \mathrm{H}-3^{\prime}\right.$, H-5'), 5.25 (s, $2 \mathrm{H}, \mathrm{CH}_{2}$ ), 4.29 (q, $\left.{ }^{3} J_{1^{\prime \prime}, 2^{\prime \prime}}=7.2 \mathrm{~Hz}, 2 \mathrm{H}, \mathrm{H}-1 "\right), 1.31$ (t, $\left.{ }^{3} J_{1^{\prime \prime}, 2^{\prime \prime}}=7.2 \mathrm{~Hz}, 3 \mathrm{H}, \mathrm{H}-2^{\prime \prime}\right)$ ppm; ${ }^{13} \mathrm{C}$ NMR (50 MHz, $\left.\mathrm{CDCl}_{3}, 25{ }^{\circ} \mathrm{C}\right): \delta=166.1(\mathrm{CO}), 158.7\left(\mathrm{~d},{ }^{1} J_{2}, \mathrm{~F}=251.0 \mathrm{~Hz}, \mathrm{C}-2^{\prime}\right)$, $140.8(\mathrm{C}-4), 128.7\left(\mathrm{~d},{ }^{3} J_{6^{\prime}, \mathrm{F}}=4.1 \mathrm{~Hz}, \mathrm{C}-6^{\prime}\right), 128.0\left(\mathrm{~d},{ }^{4} J_{5^{\prime}, \mathrm{F}}=3.6 \mathrm{~Hz}, \mathrm{C}-5^{\prime}\right), 124.2\left(\mathrm{~d},{ }^{4} J_{5, \mathrm{~F}}=13.0\right.$ $\mathrm{Hz}, \mathrm{C}-5), 121.8\left(\mathrm{~d},{ }^{3} J_{4^{\prime}, \mathrm{F}}=9.5 \mathrm{~Hz}, \mathrm{C}-4^{\prime}\right), 119.3\left(\mathrm{~d},{ }^{2} J_{3^{\prime}, \mathrm{F}}=24.6 \mathrm{~Hz}, \mathrm{C}-3^{\prime}\right), 117.4\left(\mathrm{~d},{ }^{2} J_{1^{\prime}, \mathrm{F}}=12.8\right.$ $\left.\mathrm{Hz}, \mathrm{C}-1^{\prime}\right), 62.5\left(\mathrm{CH}_{2}\right), 50.9$ (C-1"), 14.0 (C-2") ppm. ESI-MS: m/z (\%) = 329 (35), 327 (35) $\left[\mathrm{M}^{+}\right] ; 229$ (51), 227 (61) $\left[\mathrm{M}^{+}-100\right] ; 214$ (100), 212 (68) [ $\left.\mathrm{M}^{+}-115\right]$. Anal. Calcd. for $\mathrm{C}_{12} \mathrm{H}_{11} \mathrm{BrFN}_{3} \mathrm{O}_{2}$ : C, 43.92; H, 3.38; N, 12.81. Found: C, 44.22; H, 3.45; N 12.54 .

4-(4-Chlorophenyl)-2-(ethoxycarbonyl)methyl-5-phenyl-2H-[1,2,3]triazole (17a). White solid (104.1 mg, 60\%); m.p. 94-96 ${ }^{\circ} \mathrm{C}$ (cyclohexane/AcOEt). ${ }^{1} \mathrm{H}$ NMR $\left(200 \mathrm{MHz}, \mathrm{CDCl}_{3}, 25\right.$ $\left.{ }^{\circ} \mathrm{C}\right): \delta=7.54-7.47\left(\mathrm{~m}, 4 \mathrm{H}, \mathrm{H}_{o}, \mathrm{H}_{o}\right), 7.38-7.35\left(\mathrm{~m}, 3 \mathrm{H}, \mathrm{H}_{m}, \mathrm{H}_{p^{\prime}}\right), 7.32\left(\mathrm{dt},{ }^{3} J_{\mathrm{o}, \mathrm{m}}=8.8 \mathrm{~Hz},{ }^{4} J_{\mathrm{m}, \mathrm{m}}=\right.$ $\left.{ }^{5} J_{\mathrm{o}, \mathrm{m}}=2.2 \mathrm{~Hz}, 2 \mathrm{H}, \mathrm{H}_{m}\right), 5.17\left(2 \mathrm{H}, \mathrm{s}, \mathrm{CH}_{2}\right), 4.19\left(\mathrm{q},{ }^{3} J_{1^{\prime}, 2^{\prime}}=7.0 \mathrm{~Hz}, 2 \mathrm{H}, \mathrm{H}-1\right.$ ') $1.21\left(\mathrm{t},{ }^{3} J_{1^{\prime}, 2^{\prime}}=\right.$ $\left.7.0 \mathrm{~Hz}, 3 \mathrm{H}, \mathrm{H}-2^{\prime}\right)$ ppm; ${ }^{13} \mathrm{C}$ NMR $\left(50 \mathrm{MHz}, \mathrm{CDCl}_{3}, 25^{\circ} \mathrm{C}\right): \delta 166.6(\mathrm{CO}), 145.6(\mathrm{C}-5), 144.4(\mathrm{C}-$ 4), $\left.134.4\left(C_{p}\right), 130.4\left(\mathrm{C}_{i p s o}\right)^{\prime}\right), 129.5\left(\mathrm{C}_{o}\right), 129.1\left(\mathrm{C}_{i p s o}\right), 128.7\left(\mathrm{C}_{m}\right), 128.6\left(\mathrm{C}_{p^{\prime}}, \mathrm{C}_{m^{\prime}}\right), 128.3\left(\mathrm{C}_{o^{\prime}}\right)$, $62.1\left(\mathrm{CH}_{2}\right), 55.6\left(\mathrm{C}-1^{\prime}\right), 14.1\left(\mathrm{C}-2^{\prime}\right) \mathrm{ppm}$. ESI-MS: $\mathrm{m} / \mathrm{z}(\%)=343(41), 341(95)\left[\mathrm{M}^{+}\right] ; 270(45)$, $268(100)\left[\mathrm{M}^{+}-73\right] ; 104(50)\left[\mathrm{M}^{+}-237\right]$. Anal. Calcd. for $\mathrm{C}_{18} \mathrm{H}_{16} \mathrm{ClN}_{3} \mathrm{O}_{2}$ : C, 63.25; H, 4.72; N, 12.29. Found: C, 62.99; H, 5.01; N 12.08 .

\section{Synthesis of compounds 18-23. General procedure}

To a solution of amine $(0.75 \mathrm{mmol})$ in dry $\mathrm{CH}_{2} \mathrm{Cl}_{2}(0.50 \mathrm{~mL})$ was added dropwise under nitrogen a solution of trimethylaluminium in heptane $2 \mathrm{M}(0.38 \mathrm{~mL}, 0.75 \mathrm{mmol})$. The mixture was stirred at room temperature for $1 \mathrm{~h}$. 16a-17a $(0.3 \mathrm{mmol})$ in dry $\mathrm{CH}_{2} \mathrm{Cl}_{2}(0.5 \mathrm{~mL})$ was added under nitrogen and the solution was warmed to $40^{\circ} \mathrm{C}$ for $2 \mathrm{~h}$. The reaction mixture was raised to $0{ }^{\circ} \mathrm{C}$ and poured carefully (exothermic reaction) into $\mathrm{HCl}(2 \mathrm{~N}, 4.68 \mathrm{~mL})$. The resultant mixture was stirred at $40^{\circ} \mathrm{C}$ for $0.5 \mathrm{~h}$. The aqueous layer was then extracted with $3 \times 10 \mathrm{~mL} \mathrm{CH}_{2} \mathrm{Cl}_{2}$. The combined organic extracts were dried over $\mathrm{Na}_{2} \mathrm{SO}_{4}$ and the oily residue was purified by column chromatography eluting with cyclohexane/AcOEt (1:1). RMN signals of 18-21 were described to majority conformer.

4-(4-Chlorophenyl)-5-phenyl-2-(piperidin-1-yl-carbamoyl)methyl-2H-[1,2,3]triazole (18). White solid (45.1 mg, 38\%); m.p. 204-206 ${ }^{\circ} \mathrm{C}$ (toluene). ${ }^{1} \mathrm{H}$ NMR $\left(500 \mathrm{MHz}, \mathrm{DMSO}-\mathrm{d}_{6}, 25^{\circ} \mathrm{C}\right)$ : $\delta=9.03(\mathrm{~s}, 1 \mathrm{H}, \mathrm{NH}), 7.49-7.38\left(\mathrm{~m}, 9 \mathrm{H}, \mathrm{H}_{\mathrm{ar}}\right), 5.46\left(\mathrm{~s}, 2 \mathrm{H}, \mathrm{CH}_{2}\right), 3.02$ (sw, $2 \mathrm{H}, \mathrm{H}-2 \mathrm{ec}$ ) $), 2.71-$ 2.69 (m, 2 H, H-2ax'), 2.40 (sw, 1 H, H-4ec'), 1.56-1.52 (m, 5 H, H-3', H-4ax') ppm; ${ }^{13} \mathrm{C}^{\mathrm{N} M R}$ (100 MHz, DMSO-d $\left.6,25{ }^{\circ} \mathrm{C}\right): \delta=168.1(\mathrm{CO}), 144.6(\mathrm{C}-5), 143.3(\mathrm{C}-4), 133.8\left(C_{p}\right), 131.1$ $\left(\mathrm{C}_{i p s o^{\prime}}\right), 130.2\left(\mathrm{C}_{o}\right), 129.5\left(\mathrm{C}_{p^{\prime}}, \mathrm{C}_{m}, \mathrm{C}_{m^{\prime}}\right), 129.3\left(\mathrm{C}_{\text {ipso }}\right), 128.6\left(\mathrm{C}_{o^{\prime}}\right), 57.3\left(\mathrm{C}-2^{\prime}\right), 56.2\left(\mathrm{CH}_{2}\right), 26.0$ 


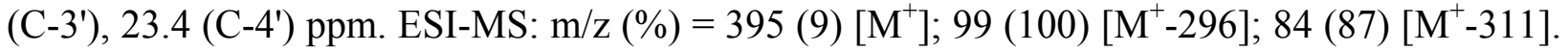
Anal. Calcd. for $\mathrm{C}_{21} \mathrm{H}_{22} \mathrm{ClN}_{5} \mathrm{O}: \mathrm{C}, 63.71 ; \mathrm{H}, 5.60 ; \mathrm{N}, 17.69$. Found: C, 63.43; H, 6.00; N 17.24.

4-(4-Bromo-2-fluorophenyl)-2-(piperidin-1-yl-carbamoyl)methyl-2H-[1,2,3]triazole (19). White solid (50.5 mg, 44\%); m.p. 235-237 ${ }^{\circ} \mathrm{C}$ (toluene). $\left.{ }^{1} \mathrm{H} \mathrm{NMR} \mathrm{(400} \mathrm{MHz,} \mathrm{CDCl}_{3}, 25^{\circ} \mathrm{C}\right): \delta=$ $8.01\left(\mathrm{~d},{ }^{5} J_{5, \mathrm{~F}}=2.1 \mathrm{~Hz}, 1 \mathrm{H}, \mathrm{H}-5\right), 7.87\left(\mathrm{t},{ }^{3} J_{6^{\prime}, 5^{\prime}}={ }^{4} J_{6^{\prime}, \mathrm{F}}=8.3 \mathrm{~Hz}, 1 \mathrm{H}, \mathrm{H}-66^{\prime}\right), 7.32\left(\mathrm{~d},{ }^{3} J_{3^{\prime}, \mathrm{F}}=8.3\right.$ $\mathrm{Hz},{ }^{3} J_{5^{\prime}, 6^{\prime}}=8.3 \mathrm{~Hz}, 2 \mathrm{H}, \mathrm{H}-3$ ', H-5'), 6.35 (sw, $\left.1 \mathrm{H}, \mathrm{NH}\right), 5.45$ (s, $2 \mathrm{H}, \mathrm{CH}_{2}$ ), 3.12 (sw, $2 \mathrm{H}, \mathrm{H}-$ 2ec"), 2.68 (sw, 1 H, H-4ec"), 2.34 (sw, 2 H, H-2ax"), 1.72-1.63 (m, 5 H, H-3", H-4ax") ppm; ${ }^{13} \mathrm{C} \mathrm{NMR}\left(100 \mathrm{MHz}, \mathrm{CDCl}_{3}, 25{ }^{\circ} \mathrm{C}\right): \delta=167.9(\mathrm{CO}), 159.5\left(\mathrm{~d},{ }^{1} J_{2^{\prime}, \mathrm{F}}=254.0 \mathrm{~Hz}, \mathrm{C}-2^{\prime}\right), 142.1(\mathrm{C}-$ 4), $134.5\left(\mathrm{~d},{ }^{4} J_{5, \mathrm{~F}}=11.4 \mathrm{~Hz}, \mathrm{C}-5\right), 129.4\left(\mathrm{~d},{ }^{3} J_{6^{\prime}, \mathrm{F}}=4.6 \mathrm{~Hz}, \mathrm{C}-6\right.$ '), $127.9\left(\mathrm{~d},{ }^{4} J_{5^{\prime}, \mathrm{F}}=3.1 \mathrm{~Hz}, \mathrm{C}-5^{\prime}\right)$, $122.1\left(\mathrm{~d},{ }^{3} J_{4^{\prime}, \mathrm{F}}=9.9 \mathrm{~Hz}, \mathrm{C}-4\right.$ '), $119.6\left(\mathrm{~d},{ }^{2} J_{3^{\prime}, \mathrm{F}}=25.2 \mathrm{~Hz}, \mathrm{C}-3^{\prime}\right), 117.5$ (d, $\left.{ }^{2} J_{1^{\prime}, \mathrm{F}}=13.0 \mathrm{~Hz}, \mathrm{C}-1^{\prime}\right)$,

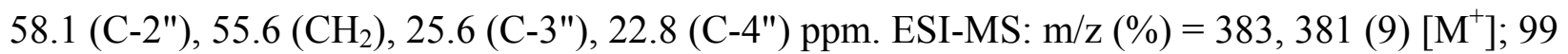
(100) $\left[\mathrm{M}^{+}-283\right] ; 83$ (92) $\left[\mathrm{M}^{+}-299\right]$. Anal. Calcd. for $\mathrm{C}_{15} \mathrm{H}_{17} \mathrm{BrFN}_{5} \mathrm{O}$ : C, 47.13; H, 4.48; N, 18.32. Found: C, 46.97; H, 4.77; N 18.18.

4-(4-Chlorophenyl)-5-phenyl-2-(morpholin-4-yl-carbamoyl)methyl-2H-[1,2,3] triazole (20). White solid (83.5 mg, 70\%); m.p. $230-232{ }^{\circ} \mathrm{C}$ (toluene). ${ }^{1} \mathrm{H}$ NMR (500 MHz, DMSO- $\left.d_{6}, 25{ }^{\circ} \mathrm{C}\right)$ : $\delta=9.15(\mathrm{~s}, 1 \mathrm{H}, \mathrm{NH}), 7.49-7.42\left(\mathrm{~m}, 9 \mathrm{H}, \mathrm{H}_{\mathrm{ar}}\right), 5.55\left(\mathrm{~s}, 2 \mathrm{H}, \mathrm{CH}_{2}\right), 3.77-3.52(\mathrm{~m}, 4 \mathrm{H}, \mathrm{H}-3$ '), 2.952.63 (m, 4 H, H-2') ppm; ${ }^{13} \mathrm{C}$ NMR (100 MHz, DMSO- $\left.d_{6}, 25^{\circ} \mathrm{C}\right): \delta=167.6(\mathrm{CO}), 143.9(\mathrm{C}-5)$, $142.6(\mathrm{C}-4), 133.1\left(\mathrm{C}_{p}\right), 130.4\left(\mathrm{C}_{i p s o}\right), 129.5\left(\mathrm{C}_{p^{\prime}}, \mathrm{C}_{o}\right), 128.9\left(\mathrm{C}_{m}\right), 128.8\left(\mathrm{C}_{m^{\prime}}\right), 128.6\left(\mathrm{C}_{i p s o}\right)$, $127.9\left(\mathrm{C}_{o^{\prime}}\right), 65.9\left(\mathrm{C}-3^{\prime}\right), 55.7\left(\mathrm{C}-2^{\prime}\right), 55.5\left(\mathrm{CH}_{2}\right) \mathrm{ppm}$. ESI-MS: m/z (\%) = 399 (14), 397 (39)

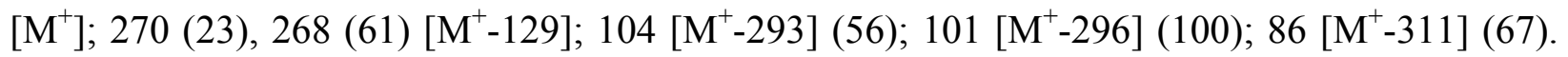
Anal. Calcd. for $\mathrm{C}_{20} \mathrm{H}_{20} \mathrm{ClN}_{5} \mathrm{O}_{2}$ : C, 60.38; H, 5.07; N, 17.60. Found: C, 60.15; H, 5.31; N 17.43.

4-(4-Bromo-2-fluorophenyl)-2-(morpholin-4-yl-carbamoyl)methyl-2H-[1,2,3]triazole (21). White solid (73.8 mg, 64\%); m.p. $254-256{ }^{\circ} \mathrm{C}$ (toluene). ${ }^{1} \mathrm{H}$ NMR (500 MHz, DMSO- $\left.d_{6}, 25{ }^{\circ} \mathrm{C}\right)$ : $\delta=9.10(\mathrm{~s}, 1 \mathrm{H}, \mathrm{NH}), 8.16\left(\mathrm{~d},{ }^{5} J_{5, \mathrm{~F}}=3.3 \mathrm{~Hz}, 1 \mathrm{H}, \mathrm{H}-5\right), 7.85\left(\mathrm{dd},{ }^{3} J_{6^{\prime}, 5^{\prime}}=8.3 \mathrm{~Hz},{ }^{4} J_{6^{\prime}, \mathrm{F}}=7.8 \mathrm{~Hz}\right.$, $\left.1 \mathrm{H}, \mathrm{H}-6^{\prime}\right), 7.73\left(\mathrm{dd},{ }^{3} J_{3^{\prime}, \mathrm{F}}=10.3 \mathrm{~Hz},{ }^{4} J_{3^{\prime}, 5^{\prime}}=2.0 \mathrm{~Hz}, 1 \mathrm{H}, \mathrm{H}-3^{\prime}\right), 7.53\left(\mathrm{dd},{ }^{3} J_{6^{\prime}, 5^{\prime}}=8.3 \mathrm{~Hz},{ }^{4} J_{5^{\prime}, 3^{\prime}}=\right.$ $\left.2.0 \mathrm{~Hz}, 1 \mathrm{H}, \mathrm{H}-5^{\prime}\right), 5.53$ (s, $2 \mathrm{H}, \mathrm{CH}_{2}$ ), 3.61-3.59 (m, 4 H, H-3"), 2.77-2.75 (m, 4 H, H-2") ppm; ${ }^{13} \mathrm{C}$ NMR (125 MHz, DMSO- $\left.d_{6}, 25{ }^{\circ} \mathrm{C}\right): \delta=167.5(\mathrm{CO}), 158.8\left(\mathrm{~d},{ }^{1} J_{2^{\prime}, \mathrm{F}}=253.3 \mathrm{~Hz}, \mathrm{C}-2^{\prime}\right), 140.7$ (C-4), $133.7\left(\mathrm{~d},{ }^{4} J_{5, \mathrm{~F}}=9.9 \mathrm{~Hz}, \mathrm{C}-5\right), 129.4\left(\mathrm{~d},{ }^{3} J_{6^{\prime}, \mathrm{F}}=4.1 \mathrm{~Hz}, \mathrm{C}-6 \mathrm{\prime}\right), 128.3\left(\mathrm{~d},{ }^{4} J_{5^{\prime}, \mathrm{F}}=3.1 \mathrm{~Hz}, \mathrm{C}-\right.$ $\left.5^{\prime}\right), 121.6\left(\mathrm{~d},{ }^{3} J_{4^{\prime}, \mathrm{F}}=9.8 \mathrm{~Hz}, \mathrm{C}-4^{\prime}\right), 119.7\left(\mathrm{~d},{ }^{2} J_{3^{\prime}, \mathrm{F}}=24.4 \mathrm{~Hz}, \mathrm{C}-3^{\prime}\right), 117.3\left(\mathrm{~d},{ }^{3} J_{1^{\prime}, \mathrm{F}}=13.0 \mathrm{~Hz}, \mathrm{C}-\right.$ 1'), 65.9 (C-3"), 55.7 (C-2"), $55.6\left(\mathrm{CH}_{2}\right) \mathrm{ppm}$. ESI-MS: m/z (\%) = 385, 383 (4) $\left[\mathrm{M}^{+}\right] ; 300,298$ (7) [M-85]; 256, 254 (11) [M-129]; 202, 200 (16) [M-185]; 200, 198 (16) [M-183]; 101 (100) [M-283]. Anal. Calcd. for $\mathrm{C}_{14} \mathrm{H}_{15} \mathrm{BrFN}_{5} \mathrm{O}_{2}: \mathrm{C}, 43.77 ; \mathrm{H}, 3.94 ; \mathrm{N}, 18.23$. Found: C, 43.94; H, $3.90 ;$ N 18.62.

\section{4-(4-Chlorophenyl)-5-phenyl-2-(cyclohexyl-carbamoyl)methyl-2H-[1,2,3]triazole}

(22). White solid (66.3 mg, 56\%); m.p. $192-194{ }^{\circ} \mathrm{C}$ (cyclohexane). ${ }^{1} \mathrm{H}$ NMR (500 MHz, $\mathrm{CDCl}_{3}, 25$ $\left.{ }^{\circ} \mathrm{C}\right): \delta=7.52-7.50\left(\mathrm{~m}, 2 \mathrm{H}, \mathrm{H}_{o}\right), 7.48\left(\mathrm{~d},{ }^{3} J_{\mathrm{o}, \mathrm{m}}=8.3 \mathrm{~Hz}, 2 \mathrm{H}, \mathrm{H}_{o}\right), 7.39-7.38\left(\mathrm{~m}, 3 \mathrm{H}, \mathrm{H}_{m^{\prime}}, \mathrm{H}_{p^{\prime}}\right)$ $7.33\left(\mathrm{~d},{ }^{3} J_{\mathrm{o}, \mathrm{m}}=8.3 \mathrm{~Hz}, 2 \mathrm{H}, \mathrm{H}_{m}\right), 6.14\left(\mathrm{~d},{ }^{3} J_{\mathrm{NH}, 1 \mathrm{ax}}=6.6 \mathrm{~Hz}, 1 \mathrm{H}, \mathrm{NH}\right), 5.14\left(\mathrm{~s}, 2 \mathrm{H}, \mathrm{CH}_{2}\right), 3.82$ $\left(\mathrm{tq},{ }^{3} J_{1 \mathrm{ax}, 2 \mathrm{ax} x^{\prime}}=10.2 \mathrm{~Hz},{ }^{3} J_{1 \mathrm{ax}, 2 \mathrm{eq}}={ }^{3} J_{\mathrm{NH}, 1 \mathrm{ax}}=6.6 \mathrm{~Hz}, 1 \mathrm{H}, \mathrm{H}-1 \mathrm{ax}\right), 1.88$ (m, $2 \mathrm{H}, \mathrm{H}-2 \mathrm{eq}$ '), 1.63 (m, 2 H, H-2ax'), 1.57 (m, 1 H, H-4eq'), 1.36 (m, 2 H, H-3eq'), 1.17 (m, 3 H, H-3ax', H-4ax') ppm; ${ }^{13} \mathrm{C} \mathrm{NMR}\left(50 \mathrm{MHz}, \mathrm{CDCl}_{3}, 25{ }^{\circ} \mathrm{C}\right): \delta=164.3(\mathrm{CO}), 145.8$ (C-5), $144.5(\mathrm{C}-4), 134.7\left(\mathrm{C}_{p}\right), 130.0$ 
$\left(\mathrm{C}_{i p s o^{\prime}}\right), 129.4\left(\mathrm{C}_{o}\right), 128.9\left(\mathrm{C}_{i p s o}, \mathrm{C}_{m}\right), 128.7\left(\mathrm{C}_{p^{\prime}}, \mathrm{C}_{m^{\prime}}\right), 128.2\left(\mathrm{C}_{o^{\prime}}\right), 57.7\left(\mathrm{CH}_{2}\right), 48.4\left(\mathrm{C} 1^{\prime}\right), 32.6(\mathrm{C}-$

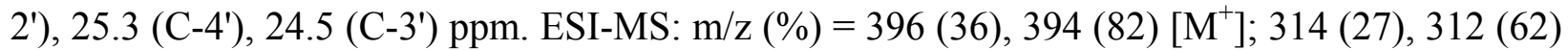

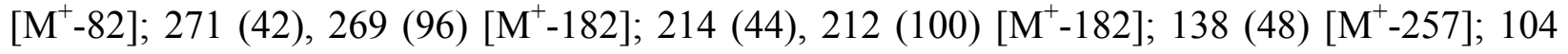
(70) $\left[\mathrm{M}^{+}-291\right]$. Anal. Calcd. for $\mathrm{C}_{22} \mathrm{H}_{23} \mathrm{ClN}_{4} \mathrm{O}: \mathrm{C}, 66.91 ; \mathrm{H}, 5.87 ; \mathrm{N}, 14.19$. Found: $\mathrm{C}, 66.82 ; \mathrm{H}$, $6.08 ; \mathrm{N} 14.02$.

4-(4-Bromo-2-fluorophenyl)-2-(cyclohexyl-carbamoyl)methyl-2H-[1,2,3]triazole (23). White solid (48.0 mg, 42\%); m.p. $228-230{ }^{\circ} \mathrm{C}$ (toluene). ${ }^{1} \mathrm{H}$ NMR (500 MHz, DMSO- $d_{6}, 25{ }^{\circ} \mathrm{C}$ ): $\delta=$ $8.25\left(\mathrm{~d},{ }^{3} J_{\mathrm{NH}, 1^{\prime \prime}}=7.5 \mathrm{~Hz}, 1 \mathrm{H}, \mathrm{NH}\right), 8.15\left(\mathrm{~d},{ }^{5} J_{5, \mathrm{~F}}=3.4 \mathrm{~Hz}, 1 \mathrm{H}, \mathrm{H}-5\right), 7.85\left(\mathrm{dd},{ }^{4} J_{6, \mathrm{~F}}=9.2 \mathrm{~Hz}\right.$, $\left.{ }^{3} J_{6^{\prime}, 5^{\prime}}=8.3 \mathrm{~Hz}, 1 \mathrm{H}, \mathrm{H}-6^{\prime}\right), 7.72\left(\mathrm{~d},{ }^{3} J_{3^{\prime}, \mathrm{F}}=10.3 \mathrm{~Hz}, 1 \mathrm{H}, \mathrm{H}-3^{\prime}\right), 7.52\left(\mathrm{~d},{ }^{3} J_{6^{\prime}, 5^{\prime}}=8.3 \mathrm{~Hz}, 1 \mathrm{H}, \mathrm{H}-5^{\prime}\right)$, 5.15 (s, $2 \mathrm{H}, \mathrm{CH}_{2}$ ), 3.53-3.52 (m, $\left.1 \mathrm{H}, \mathrm{H}-1 "\right), 1.76-1.51$ (m, $\left.4 \mathrm{H}, \mathrm{H}-2 "\right), 1.28-1.06$ (m, $6 \mathrm{H}, \mathrm{H}-3 "$, H-4"); ${ }^{13} \mathrm{C}$ NMR (125 MHz, DMSO- $\left.d_{6}, 25^{\circ} \mathrm{C}\right): \delta=164.0(\mathrm{CO}), 158.9\left(\mathrm{~d},{ }^{1} J_{2^{\prime}, \mathrm{F}}=253.3 \mathrm{~Hz}, \mathrm{C}-2^{\prime}\right)$, $140.8(\mathrm{C}-4), 133.8\left(\mathrm{~d},{ }^{4} J_{5, \mathrm{~F}}=9.9 \mathrm{~Hz}, \mathrm{C}-5\right), 129.5\left(\mathrm{~d},{ }^{3} J_{6, \mathrm{~F}}=3.8 \mathrm{~Hz}, \mathrm{C}-6{ }^{\prime}\right), 128.3\left(\mathrm{~d},{ }^{4} J_{5^{\prime}, \mathrm{F}}=3.1\right.$ $\left.\mathrm{Hz}, \mathrm{C}-5^{\prime}\right), 121.7$ (d, $\left.{ }^{3} J_{4^{\prime}, \mathrm{F}}=9.9 \mathrm{~Hz}, \mathrm{C}-4^{\prime}\right), 119.7\left(\mathrm{~d},{ }^{2} J_{3^{\prime}, \mathrm{F}}=25.2 \mathrm{~Hz}, \mathrm{C}-3^{\prime}\right), 117.2\left(\mathrm{~d},{ }^{3} J_{1^{\prime}, \mathrm{F}}=13.0\right.$ Hz, C-1'), $56.9\left(\mathrm{CH}_{2}\right), 47.8$ (C-1"), 32.3 (C-2"), 25.1 (C-4"), 24.4 (C-3") ppm. ESI-MS: m/z (\%)

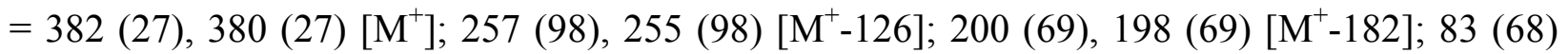
[M+283]; 55 (78) [ $\left.\mathrm{M}^{+}-327\right]$. Anal. Calcd. for $\mathrm{C}_{16} \mathrm{H}_{18} \mathrm{BrFN}_{4} \mathrm{O}$ : C, 50.41; H, 4.76; N, 14.70. Found: C, 50.72; H, 4.58; N 14.95 .

\section{Computational details}

The geometry of the systems has been fully optimized at the B3LYP/6-31G(d) computational level. ${ }^{32,33}$ Frequency calculation has been carried out at the same computational level to confirm that the structures obtained correspond to energetic minima. ${ }^{34} \mathrm{~A}$ further geometry optimization has been performed at the B3LYP/6-311++G(d,p) level. ${ }^{35}$ These geometries have been used to calculated the absolute chemical shielding within the GIAO approximation ${ }^{36}$ at the B3LYP/6$311++\mathrm{G}(\mathrm{d}, \mathrm{p})$ computational level. All these calculations were carried out using the facilities of Gaussian $03 .{ }^{37}$

\section{Acknowledgements}

PG, NJ and CGO are grateful to the Spanish National Health Program (SAF2006-13391-C03-02 and SAF2009-12422-C02-02) and RETICS RTA (RD06/001/0014), and the program CANNAB-CM ( S-SAL0261-2006).

\section{References}

1. Jagerovic, N.; Gómez de la Oliva, C.; Goya, P.; Dordal, A.; Cuberes, R. Eur. Pat. Appl. 0380 290, 2006.

2. Graham, E. S.; Ashton, J. C.; Glass, M. Front. Biosci. 2009, 14, 944. 
3. Jagerovic, N.; Fernandez-Fernandez, C.; Goya, P. Curr. Top. Med. Chem. 2008, 8, 205.

4. Marriott, K. S.; Huffman, J. W. Curr. Top. Med. Chem. 2008, 8, 187.

5. Viveros, M. P.; de Fonseca, F. R.; Bermudez-Silva, F. J.; McPartland, J. M. Endocr. Metab. Immune Disord. Drug Targets 2008, 8, 220.

6. Di Marzo, V. Drug Discov. Today 2008, 13, 1026.

7. Le Foll, B.; Gorelick, D.A.; Goldberg, S.R. Psychopharmacology 2009, 205, 171.

8. Jagerovic, N.; Hernández-Folgado, L.; Alkorta, I.; Goya, P.; Navarro, M.; Serrano, A.; Rodríguez de Fonseca, F.; Dannert, M.T.; Alsasua, A.; Suardiaz, M.; Pascual, D.; Martín, M. I. J. Med. Chem. 2004, 47, 2939.

9. Pavón, F. J.; Bilbao, A.; Hernández-Folgado, L.; Cippitelli, A.; Jagerovic, N.; Abellan, G.; Rodríguez-Franco, M. I.; Serrano, A.; Macias, M.; Gómez, R.; Navarro, M.; Goya, P.; Rodríguez de Fonseca, F. Neuropharmacology 2006, 51, 358.

10. Pavón, F. J.; Serrano, A.; Pérez-Valero, V.; Jagerovic, N.; Hernandez-Folgado, L.; Bermudez-Silva, F.J.; Macias, M.; Goya, P; de Fonseca, F. R. J. Neuroendocrinol. 2008, 20, 116.

a. Appendino, G.; Bacchiega, S.; Minassi, A.; Cascio, M. G.; De Petrocellis, L.; Di Marzo V. Angew. Chem. Int. Ed. 2007, 46, 9312.

11. Shu, H.; Izenwasser, S.; Wade, D.; Stevens, E. D; Trudell, M. L. Bioorg. Med. Chem. Lett. 2009, 19, 891 .

12. Hou, D.-R.; Alam, S.; Kuan, T.-C.; Ramanathan, M.; Lin, T.-P.; Hung, M.-S. Bioorg. Med. Chem. Lett. 2009, 19, 1022.

13. Tomé, A. C. Sci. Synth. 2004, 13, 415.

14. Huisgen, R. J. Org. Chem. 1976, 41, 403.

15. Huisgen, R.; Knorr, R.; Mobius, L.; Szeimies, G. Chem. Ber. 1965, 98, 4014.

16. Kolb, J. H. C.; Sharpless, K. B. Drug Discov. Today 2003, 8, 1128.

17. Zhang, L.; Chen, X. G.; Xue, P.; Sun, H. H. Y.; Williams, I. D.; Sharpless, K. B.; Fokin, V. V.; Jia, G. C. J. Am. Chem. Soc. 2005, 127, 15998.

18. Kalisiak, J.; Sharpless, K. B.; Fokin, V. V. Org. Lett. 2008, 10, 3171.

19. Kamijo, S.; Jin, T.; Huo, Z.; Yamamoto, Y. J. Am. Chem. Soc. 2003, 125, 7786.

20. Barral, K.; Moorhouse, A. D.; Moses, J. E. Org. Lett. 2007, 9, 1809.

21. Tang, W. J., Hu, Y. Z. Synth. Commun. 2006, 36, 2461.

22. Krivopalov V. P.; Shkurko O. P. L. Russ. Chem. Rev. 2005, 74, 339.

23. Hitomi, T.; Kozima, S. J. Organomet. Chem. 1977, 127, 273.

24. Nguefack, J. F.; Bolitt, V., Sinou, D. Tetrahedron Lett. 1996, 37, 5527.

25. Pardo, C; Graf, S.; Ramos, M.; Sesmilo, E.; Elguero, J. Org. Prep. Proced. Int. 2000, 32, 385.

26. I. Alkorta, J. Elguero, Computational NMR Spectroscopy, in J. Grunenberg, Ed. 2010, in press.

27. Blanco, F.; Alkorta, I.; Elguero, J. Magn. Reson. Chem. 2007, 45, 797. 
28. Silva, A. M. S.; Sousa, R. M. S.; Jimeno, M. L.; Blanco, F.; Alkorta, I.; Elguero, J. Magn.Reson. Chem. 2008, 46, 859.

29. Jagerovic N.; Hernandez-Folgado, L.; Alkorta, I.; Goya, P.; Martín, M. I.; Dannert, M. T.; Alsasua, A.; Frigola, J.; Cuberes, M. R.; dordal, A.; Holenz, J. Eu. J. Med. Chem. 2006, 41, 114.

30. Dugave, C.; Demange, L. Chem. Rev. 2003, 103, 2475.

31. Becke, A. D. Phys. Rev. A 1988, 38, 3098. Becke, A. D. J. Chem. Phys. 1993, 98, 5648. Lee, C.; Yang, W.; Parr, R. G. Phys. Rev. B 1988, 37, 785.

32. Hariharan, P. A.; Pople, J. A. Theor. Chim. Acta 1973, 28, 213.

33. McIver, J. W.; Komornicki, A. K. J. Am. Chem. Soc. 1972, 94, 2625.

34. Ditchfield, R.; Hehre, W. J.; Pople, J. A. J. Chem. Phys. 1971, 54, 724. Frisch, M. J.; Pople, J. A.; Krishnam, R.; Binkley, J. S. J. Chem. Phys. 1984, 80, 3265.

35. Ditchfield, R. Mol. Phys. 1974, 27, 789. London, F. J. Phys. Radium 1937, 8, 397.

36. Frisch, M. J.; Trucks, G. W.; Schlegel, H. B.; Scuseria, G. E.; Robb, M. A.; Cheeseman, J. R.; Montgomery, Jr., J. A.; Vreven, T.; Kudin, K. N.; Burant, J. C.; Millam, J. M.; Iyengar, S. S.; Tomasi, J.; Barone, V.; Mennucci, B.; Cossi, M.; Scalmani, G.; Rega, N.; Petersson, G. A.; Nakatsuji, H.; Hada, M.; Ehara, M.; Toyota, K.; Fukuda, R.; Hasegawa, J.; Ishida, M.; Nakajima, T.; Honda, Y.; Kitao, O.; Nakai, H.; Klene, M.; Li, X.; Knox, J. E.; Hratchian, H. P.; Cross, J. B.; Adamo, C.; Jaramillo, J.; Gomperts, R.; Stratmann, R. E.; Yazyev, O.; Austin, A. J.; Cammi, R.; Pomelli, C.; Ochterski, J. W.; Ayala, P. Y.; Morokuma, K.; Voth, G. A.; Salvador, P.; Dannenberg, J. J.; Zakrzewski, V. G.; Dapprich, S.; Daniels, A. D.; Strain, M. C.; Farkas, O.; Malick, D. K.; Rabuck, A. D.; Raghavachari, K.; Foresman, J. B.; Ortiz, J. V.; Cui, Q.; Baboul, A. G.; Clifford, S.; Cioslowski, J.; Stefanov, B. B.; Liu, G.; Liashenko, A.; Piskorz, P.; Komaromi, I.; Martin, R. L.; Fox, D. J.; Keith, T.; Al-Laham, M. A.; Peng, C. Y.; Nanayakkara, A.; Challacombe, M.; Gill, P. M. W.; Johnson, B.; Chen, W.; Wong, M. W.; Gonzalez, C. and Pople, J. A. Gaussian 03, Gaussian, Inc., Pittsburgh PA, 2003. 